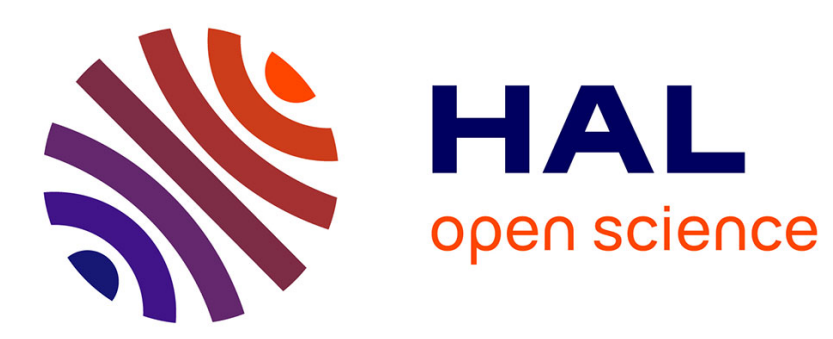

\title{
Self-averaging and weak ergodicity breaking of diffusion in heterogeneous media
}

\author{
A Russian, Marco Dentz, Philippe Gouze
}

\section{To cite this version:}

A Russian, Marco Dentz, Philippe Gouze. Self-averaging and weak ergodicity breaking of diffusion in heterogeneous media. Physical Review E , 2017, 96 (2), pp.022156. 10.1103/PhysRevE.96.022156 . hal-01622668v2

\section{HAL Id: hal-01622668 \\ https://hal.science/hal-01622668v2}

Submitted on 24 Oct 2017

HAL is a multi-disciplinary open access archive for the deposit and dissemination of scientific research documents, whether they are published or not. The documents may come from teaching and research institutions in France or abroad, or from public or private research centers.
L'archive ouverte pluridisciplinaire HAL, est destinée au dépôt et à la diffusion de documents scientifiques de niveau recherche, publiés ou non, émanant des établissements d'enseignement et de recherche français ou étrangers, des laboratoires publics ou privés. 


\title{
Self-averaging and weak ergodicity breaking of diffusion in heterogeneous media
}

\author{
Anna Russian* \\ Dipartimento di Ingegneria Civile e Ambientale, Politecnico di Milano, Milano, Italy \\ Marco Dentz ${ }^{\dagger}$ \\ Spanish National Research Council (IDAEA-CSIC), 08034 Barcelona, Spain \\ Philippe Gouze \\ Géosciences, Université de Montpellier, CNRS, Montpellier, France
}

(Received 26 May 2017; revised manuscript received 13 July 2017; published 30 August 2017)

\begin{abstract}
Diffusion in natural and engineered media is quantified in terms of stochastic models for the heterogeneityinduced fluctuations of particle motion. However, fundamental properties such as ergodicity and self-averaging and their dependence on the disorder distribution are often not known. Here, we investigate these questions for diffusion in quenched disordered media characterized by spatially varying retardation properties, which account for particle retention due to physical or chemical interactions with the medium. We link self-averaging and ergodicity to the disorder sampling efficiency $R_{n}$, which quantifies the number of disorder realizations a noise ensemble may sample in a single disorder realization. Diffusion for disorder scenarios characterized by a finite mean transition time is ergodic and self-averaging for any dimension. The strength of the sample to sample fluctuations decreases with increasing spatial dimension. For an infinite mean transition time, particle motion is weakly ergodicity breaking in any dimension because single particles cannot sample the heterogeneity spectrum in finite time. However, even though the noise ensemble is not representative of the single-particle time statistics, subdiffusive motion in $q \geqslant 2$ dimensions is self-averaging, which means that the noise ensemble in a single realization samples a representative part of the heterogeneity spectrum.
\end{abstract}

DOI: 10.1103/PhysRevE.96.022156

\section{INTRODUCTION}

The diffusion of particles in a fluid or gas at rest is described through Brownian motion, characterized by a linear increase of the mean-square displacement and Gaussian displacement statistics. Fick's laws form the basis for the quantification of tracer motion and chemical interactions between diffusing species. These mechanisms, however, are very different for diffusion in heterogeneous media, in which particle motion may be hindered due to spatial variability in the medium properties $[1,2]$. In fact, anomalous diffusion is a ubiquitous phenomenon [1,3-5], observed in natural and engineered media, which are intrinsically heterogeneous. Diffusion in heterogeneous media refers to processes as diverse as radionuclide migration in low-permeability geological media [6-10], surface and solid-state diffusion [11-13], natural gas production from tight reservoirs [14-16], contaminant and heat transfer in the subsurface [17-21], the motion of charge carriers in amorphous semiconductors [22], and the transmission of light in optical media [23], as well as the motion of endogenous and artificial tracers in living cells and cell membranes [24-29].

Stochastic modeling of particle motion in quenched random systems is particularly challenging because particles sample the disorder only by diffusion, whose efficiency in turn is affected by the medium properties. The fundamental assumption underlying the use of stochastic models for the prediction of particle motion is that the ensemble behavior may be representative for the behavior in a single realization.

\footnotetext{
*anna.russian@polimi.it

${ }^{\dagger}$ marco.dentz@csic.es
}

This may refer to the representation of the temporal statistics of a single-particle trajectory through a noise ensemble, or to the representation of particle statistics in a single-medium realization through the disorder ensemble statistics. The former relates to the notion of ergodicity [30], the latter to the notion of self-averaging [1].

Self-averaging can only apply to infinite systems, which are characterized by statistically stationary disorder distributions so that diffusing particles have the possibility to sample a representative part of the disorder spectrum in a single realization. The lack of self-averaging for finite size quenched trap models has been recently discussed by Akimoto et al. [31]. Infinity of space and statistical stationarity, however, are only necessary conditions for self-averaging. It critically depends on the sampling efficiency and thus on the diffusion process, which in turn is affected by the heterogeneity [32]. Despite its fundamental nature, only few studies address the problem of self-averaging in $q$-dimensional quenched random media, specifically the evolution of the variance of the mean square displacement, for diffusive and subdiffusive systems.

Motivated by experimental results on single-particle tracking, the ergodicity of particle motion in fluctuating environments has received considerable attention [27,30,33,34] with specific focus on subdiffusive motion [34-38]. Weak ergodicity breaking in disordered media is related to long waiting times between particle transitions [33], which impedes significant disorder sampling in finite time. The latter has put the continuous time random walk (CTRW) [19,30,39] in the spotlight as a model for subdiffusive particle motion. The CTRW renews particle waiting times at each random walk step independently and thus can be seen as an annealed disorder model. Subdiffusion in the CTRW is weakly ergodicity 
breaking as a consequence of aging, this means nonstationarity of particle displacement caused by diverging particle waiting times $[35,40]$. For the same reason, subdiffusion in quenched random trap models, which show CTRW behavior in $q>2$ dimensions, has been found to be weakly ergodicity breaking [38,41]. Bertin and Bouchaud [42] analyze weak ergodicity breaking and localization of subdiffusion in the $q=1$-dimensional quenched random trap model. Massignan et al. [26] study ergodicity breaking of subdiffusion in a $q=1$-dimensional inhomogeneous environment. Only few works address the ergodicity in $q \geqslant 1$-dimensional quenched disorder under diffusive and subdiffusive conditions despite its central importance for the understanding of diffusion in heterogeneous media.

In this paper we study self-averaging and ergodicity for diffusion in $q$-dimensional quenched random media. Diffusion is hindered due to spatially varying retardation properties, which account for physical and chemical interactions with the medium. This disorder model is equivalent to the quenched random trap model [1,32]. The nature of average particle motion in the quenched random trap model depends on the dimensionality $q$ of space [1], which is strongly related to the notion of the average number $S_{n}$ of distinct sites visited as a function of step number $n$ of the random walk. The latter determines the efficiency of the diffusion process for disorder sampling in a single medium realization. For $q>2$, average particle motion describes a CTRW because particles do in average not return to the same site that has already been visited. For $q<2$ this is different. Particle motion describes a CTRW-like behavior, which however is characterized by dependence of subsequent waiting times because particles may return to the same site with finite probability. Clearly, this property has an impact on the sampling efficiency and thus self-averaging property.

This paper extends previous work [32,38], which focused on the questions of self-averaging and weak ergodicity breaking for disorder scenarios leading to subdiffusive average behaviors. Here, we derive a general framework for the analysis and quantification of self-averaging and ergodicity for arbitrary disorder distributions, characterized by diffusive and subdiffusive average behaviors. We study particle motion in single-disorder realizations to illustrate the impact of medium heterogeneity on the segregation of particle distributions and their sample to sample fluctuations. Ergodicity and selfaveraging are quantified systematically in terms of the noise variance of the time-averaged mean-square displacement in single-medium realizations (ergodicity), and the disorder variance of the noise mean-squared displacement (self-averaging). We derive analytical expressions for the disorder variance of the mean square displacement and the noise variance of the time-averaged mean-square displacement under both diffusive and subdiffusive conditions. Note that the concepts of ergodicity and self-averaging are different. Ergodicity refers to the equivalence between temporal average and noise average, this means average over all particles in a given disorder realization. Self-averaging on the other hand denotes the property that the mean square displacement, defined as a noise average in a single realization, may be represented by its disorder average. Under self-averaging conditions, the mean square displacement is asymptotically independent of the specific disorder realization. Thus, it may be weakly ergodicity breaking but self-averaging.

The paper is organized as follows. Section II describes the diffusion problem, presents the coarse-graining procedure, and derives explicit expressions for the noise average, a central part of the paper. Furthermore, it defines the disorder average in terms of the coarse grained particle trajectories. Section III discusses the disorder scenarios under consideration and illustrates the behavior of the mean square displacement and particle distributions in the average over all disorder realizations as well as in single-disorder realizations. Section IV analyzes self-averaging for different heterogeneity scenarios and derives explicit expressions for the variance of the mean square displacement. Section V discusses the ergodicity of single-particle trajectories in terms of the noise variance of the time-averaged mean-square displacement.

\section{DIFFUSION IN HETEROGENEOUS MEDIA}

Particle transport in a $q$-dimensional quenched random medium, characterized by a spatially random diffusion coefficient $D(\mathbf{x})$, can be described by the Langevin equation,

$$
\frac{d \mathbf{x}(t)}{d t}=\sqrt{2 D[\mathbf{x}(t)]} \xi(t)
$$

which is characterized by a multiplicative noise. We employ here and in the following the Ito interpretation. The Gaussian white noise has zero mean and covariance $\left\langle\xi_{i}(t) \xi_{j}\left(t^{\prime}\right)\right\rangle=$ $\delta_{i j} \delta\left(t-t^{\prime}\right)$. The angular brackets denote the noise average over all particles. The particle distribution $p(\mathbf{x}, t)=\langle\delta[\mathbf{x}-\mathbf{x}(t)]\rangle$ is then transported according to the Fokker-Planck equation [43],

$$
\frac{\partial p(\mathbf{x}, t)}{\partial t}-\nabla^{2} D(\mathbf{x}) p(\mathbf{x}, t)=0 .
$$

The diffusion coefficient in Eq. (2) is given by $D(\mathbf{x})=$ $\kappa \theta(\mathbf{x})^{-1}$, where $\kappa$ is the constant molecular diffusion coefficient. The quenched retardation coefficient $\theta(\mathbf{x})$ accounts for particle retention due to physical and chemical interactions with the medium and thus represents the medium heterogeneity. Equation (2) describes diffusion under spatially variable retardation properties. The retardation coefficient is modeled here as a stationary spatial random field which is characterized by the characteristic correlation scale $\ell$ and the distribution $p_{\theta}(\theta)$ of point values $\theta(\mathbf{x})$. Note that we consider here two stochastic processes. The temporal stochastic process $\xi(t)$, which defines the noise ensemble, and the spatial stochastic process $\theta(\mathbf{x})$ which defines the disorder ensemble. We consider in the following deterministic initial conditions of instantaneous particle injection at $t=0$ at $\mathbf{x}=\mathbf{0}$. As discussed in the Introduction and also below, particles sample the medium heterogeneity, which is represented by the disorder ensemble, due to diffusion, which is represented by the noise ensemble. Note that under certain experimental conditions the initial particle position in a single-disorder realization may be uncertain and an additional average over the initial particle locations may be required. In such a case, the noise ensemble samples part of the heterogeneity from the beginning. On the other hand, there may be situations, for example leachage of a contaminant in an underground repository, for which the initial 
positions are well confined and thus may be denominated deterministic.

The mean-square displacement in a single-medium realization is defined by $m(t)=\left\langle\mathbf{x}(t)^{2}\right\rangle$. From Eq. (1), we obtain

$$
m(t)=2 \int_{0}^{t} d t^{\prime} \int_{0}^{t} d t^{\prime \prime}\left\langle\sqrt{D\left[\mathbf{x}\left(t^{\prime}\right)\right] D\left[\mathbf{x}\left(t^{\prime \prime}\right)\right]} \xi\left(t^{\prime}\right) \cdot \xi\left(t^{\prime \prime}\right)\right\rangle .
$$

Using the Ito interpretation of the stochastic integral, we obtain the compact expression

$$
m(t)=2 q \int_{0}^{t} d t^{\prime}\left\langle D\left[\mathbf{x}\left(t^{\prime}\right)\right]\right\rangle
$$

because $\langle D[\mathbf{x}(t)] \xi(t)\rangle=0$. We set now $D(\mathbf{x})=\kappa \theta(\mathbf{x})^{-1}$ and define

$$
d s=d t \theta[\mathbf{x}(t)]^{-1} .
$$

Thus, we can write $m(t)$ in the form

$$
m(t)=2 \kappa q\langle s(t)\rangle .
$$

In the following, we use the variable transform $t \rightarrow s$ as the basis for the coarse-graining of the Langevin Eq. (1).

\section{A. Coarse graining}

The Langevin Eq. (1) can be written in the Ito interpretation as

$$
d \mathbf{x}(t)=\sqrt{2 \kappa \theta[\mathbf{x}(t)]^{-1} d t} \boldsymbol{\eta}(t),
$$

where

$$
\eta(t)=\frac{1}{\sqrt{d t}} \int_{t}^{t+d t} d t^{\prime} \boldsymbol{\xi}\left(t^{\prime}\right)
$$

is a Gaussian random vector with zero mean and unit variance such that $\left\langle\eta_{i}(t) \eta_{j}(t)\right\rangle=\delta_{i j}$. Using the transformation (4), we can separate Eq. (6) into the two equations:

$$
d \mathbf{x}(s)=\sqrt{2 \kappa d s} \eta(s), \quad d t(s)=\theta[\mathbf{x}(s)] d s .
$$

This means we have separated the nonlinearity from the spatial particle movement and transferred it to the evolution of the particle time with $s$. The process $s(t)=\max \{s \mid t(s) \leqslant t\}$ denotes the operational time that passes to reach the clock time $t$.

It is natural to choose the correlation scale $\ell$ of $\theta(\mathbf{x})$ as the spatial coarsening scale. This gives for the coarse grained equations of motion

$$
\mathbf{x}_{n+1}=\mathbf{x}_{n}+\ell \boldsymbol{\eta}_{n+1}, \quad t_{n+1}=t_{n}+\theta\left(\mathbf{x}_{n}\right) \Delta s_{n} .
$$

The random vector $\eta_{n}$ has unit length, is always aligned with one of the axes of the coordinate system, and its ensemble mean is zero. Thus, Eq. (9) describes a random walk on a $q$-dimensional lattice. The operational time increment $\Delta s_{n}$ is the first passage time to the boundaries of a region of size $\ell$ by regular diffusion. The distribution of the first passage time $\Delta s_{n}$ is cut-off exponentially for times larger than the the characteristic diffusion time $\tau_{\kappa}=\ell^{2} /(2 q \kappa)$ over the length scale $\ell$. Thus, we approximate the first passage time PDF by the exponential distribution [44],

$$
\psi_{0}\left(\Delta s_{n}\right)=\tau_{\kappa}^{-1} \exp \left(-\Delta s_{n} / \tau_{\kappa}\right) .
$$

The transition time over the length $\ell$ is given by

$$
\tau\left(\mathbf{x}_{n}\right)=\theta\left(\mathbf{x}_{n}\right) \Delta s_{n},
$$

which is a quenched random variable. Equation (9) describes a time-domain random walk (TDRW) $[9,45,46]$, which can be seen as a spatially inhomogeneous CTRW [44,47] because the transition time depends on the particle position. The choice of the coarse-graining scale as the correlation scale of the heterogeneous retardation field $\theta(\mathbf{x})$ implies that all $\tau\left(\mathbf{x}_{n}\right)$ and $\tau\left(\mathbf{x}_{n^{\prime}}\right)$ are independent for $n \neq n^{\prime}$. This means that the distribution of $\tau\left(\mathbf{x}_{n}\right)$ can be characterized by the one-point probability density function (PDF) $\psi(\tau)$ of transition times, which is given by

$$
\psi(\tau)=\int_{0}^{\infty} d \theta \theta^{-1} p_{\theta}(\theta) \psi_{0}(\tau / \theta) .
$$

It is fully determined in terms of the distribution $p_{\theta}(\theta)$ of point values of $\theta(\mathbf{x})$. This is an important fact because it allows relating the statistics of the medium heterogeneity to average particle motion as outlined in Sec. III. With these definitions, Eq. (9) is also equivalent to the quenched random trap model [1].

The space-time trajectory of a particle diffusing in the heterogeneous medium is obtained from Eq. (9):

$$
\mathbf{x}_{n}=\sum_{k=0}^{n-1} \ell \boldsymbol{\eta}_{k}, \quad t_{n}=\sum_{k=0}^{n-1} \tau\left(\mathbf{x}_{k}\right)
$$

It is worth noticing that here the sequence of $\tau\left(\mathbf{x}_{k}\right)$ is in general not composed of independent time increments, because particles may visit the same site more than once [1], depending on the dimensionality of space. This distinguishes the TDRW (9) from a classical CTRW characterized by completely independent random time increments. This point is discussed in more detail in Sec. II C below. The particle position at time $t$ is given by $\mathbf{x}(t)=\mathbf{x}_{n_{t}}$, where $n_{t}=\max \left(n \mid t_{n} \leqslant t\right)$ denotes the number of steps needed to reach time $t$ by the time random walk in Eq. (9). Likewise, the operational time $s(t)$ to arrive at time $t$ is given by $s(t)=s_{n_{t}}$. The particle density in this coarse-grained framework is given by $p(\mathbf{x}, t)=\left\langle\delta\left(\mathbf{x}-\mathbf{x}_{n_{t}}\right)\right\rangle$. We can expand $p(\mathbf{x}, t)$ as

$$
p(\mathbf{x}, t)=\sum_{n=0}^{\infty}\left\langle\delta\left(\mathbf{x}-\mathbf{x}_{n}\right) \mathbb{I}\left(t_{n} \leqslant t<t_{n+1}\right)\right\rangle,
$$

where the indicator function $\mathbb{I}\left(t_{n} \leqslant t<t_{n+1}\right)$ is 1 if the statement in the parenthesis is true and 0 else. Furthermore, the mean-square displacement, Eq. (5), is now given in terms of the coarse grained trajectory, Eq. (9), as

$$
m(t)=\ell^{2}\left\langle n_{t}\right\rangle \text {. }
$$

\section{B. Noise average}

The average diffusion behavior and its self-averaging properties depend on the efficiency of the random walker to sample the disorder, which in turn depends on the sampling efficiency due to the random noise in single-medium realizations. It is intuitive that a random walker explores a larger number of new sites as a function of random walk steps in $q=3$ than in $q=1$ spatial dimensions. In the following, we quantify this notion. 
First, we consider the coarse-grained trajectory Eq. (13) in a single-disorder realization.

As outlined in the previous section, subsequent transition times $\tau\left(\mathbf{x}_{k}\right)$ are in general not independent because particles may return to the same position after a certain number of steps. The recurrence of the random walk may be described by the average number of independent sites visited $S_{n}[1,48]$. The number $S_{n}$ depends on the dimensionality of space in accord with Polya's theorem. For $q<2$ spatial dimensions, $S_{n} \sim n^{q / 2}$, for $q=2, S_{n}=n / \ln (n)$ and for $q>2, S_{n} \sim n$. Thus, as outlined in Bouchaud and Georges [1], the $\tau\left(\mathbf{x}_{k}\right)$ in Eq. (13) may be grouped into families of independent $\tau_{i}$ with $n / S_{n}$ members each. If all members of a family are approximated by the same value $\tau_{i}$, the trajectories for single-disorder realizations can be rewritten as

$$
\mathbf{x}_{n}=\sum_{i=1}^{n} \ell \boldsymbol{\eta}_{i}, \quad t_{n}=\sum_{i=1}^{S_{n}} \frac{n}{S_{n}} \tau_{i},
$$

where the $\tau_{i}$ are distributed according to Eq. (12). In a single-disorder realization, the set of available $\tau_{i}$ to form a replica of $t_{n}$ depends on the volume sampled by the random walkers after $n$ steps. This property and the independence of subsequent transition times are the basis for the formalization of the noise average as described below, and of the disorder ensemble average as outlined in the next section.

To determine the noise average, we consider Eq. (15) for the mean-square displacement. It can be expanded according to

$$
m(t)=\ell^{2} \sum_{n=0}^{\infty} n\left\langle\mathbb{I}\left(t_{n} \leqslant t<t_{n+1}\right)\right\rangle,
$$

where we used Eq. (14). The noise average can be written as the average over all particles released in a disorder realization, such that

$$
m(t)=\ell^{2} \sum_{n=0}^{\infty} n\left\{\lim _{N \rightarrow \infty} \frac{1}{N} \sum_{j=1}^{N} \mathbb{I}\left[t_{n}^{(j)} \leqslant t<t_{n+1}^{(j)}\right]\right\},
$$

where $t_{n}^{(j)}$ denotes the time of the $j$ th particle after $n$ steps, which is defined by Eq. (16). To simplify the sum over the particles $j$, we first note that the $t_{n}^{(j)}$ are given by the sum of waiting times $\tau_{i}$, which in turn are determined by the disorder explored by the particles. Furthermore, we note that the average number of different sites sampled by the ensemble of random walkers after $n$ steps is given by $V_{n}=(2 n)^{q / 2}$, which corresponds to the characteristic volume explored by the random walkers after $n$ steps. This means that the $t_{n}^{(j)}$ are series of $\tau_{i}$ which are sampled from the same set of $V_{n}$ statistically independent copies. Next, we recall from Eq. (16) that a replica of $t_{n}$ is formed of $S_{n}$ independent $\tau_{i}$, which are drawn from a set of $V_{n}$ independent copies. Thus, the number of copies of $t_{n}$ which can be formed from $V_{n}$ members $\tau_{i}$ is equal to the binomial coefficient $B_{n}=\left(\begin{array}{c}V_{n} \\ S_{n}\end{array}\right)$. The number of independent replica of $t_{n}$ that can be formed from $V_{n}$ members on the other hand, is equal to $R_{n}=V_{n} / S_{n}$. Consequently, the number of families with members, who are statistically independent is given by $B_{n} / R_{n}$. Then, since each of the $B_{n} / R_{n}$ families explore in average the same disorder, they are in average the same. This means that the number of independent replica of $t_{n}$ in a sample of $N$ is $R_{n}$. Thus, the sum in the curly brackets of Eq. (18) can be substituted after regrouping as

$$
\sum_{j=1}^{N} \mathbb{I}\left[t_{n}^{(j)} \leqslant t<t_{n+1}^{(j)}\right]=\sum_{k=1}^{R_{n}} \frac{N}{R_{n}} \mathbb{I}\left[t_{n}^{(k)} \leqslant t<t_{n+1}^{(k)}\right] .
$$

Equation (18) for $m(t)$ then simplifies to [41]

$$
m(t)=\ell^{2} \sum_{n=0}^{\infty} \frac{n}{R_{n}} \sum_{k=1}^{R_{n}} \mathbb{I}\left[t_{n}^{(k)} \leqslant t<t_{n+1}^{(k)}\right],
$$

where the $t_{n}^{(k)}$ are independent replica of $t_{n}$. Each replica is formed according to Eq. (16), where the $\tau_{i}$ are independent identically distributed random variables characterized by the PDF in Eq. (12). Equation (20) captures the impact of noise on the disorder sampling in a single-medium realization. In general, we obtain for the noise average of the $k$ th power $n_{t}^{k}$ of the number of steps needed to arrive at time $t$,

$$
\left\langle n_{t}^{k}\right\rangle \approx \sum_{n=0}^{\infty} \frac{n^{k}}{R_{n}} \sum_{k=1}^{R_{n}} \mathbb{I}\left[t_{n}^{(k)} \leqslant t<t_{n+1}^{(k)}\right] .
$$

Similarly, the particle density, Eq. (14), in a single realization may be approximated in terms of independent replica of $t_{n}$ as

$$
p(\mathbf{x}, t)=\sum_{n=0}^{\infty}\left\langle\delta\left(\mathbf{x}-\mathbf{x}_{n}\right)\right\rangle \frac{1}{R_{n}} \sum_{i=1}^{R_{n}} \mathbb{I}\left(t_{n}^{(i)} \leqslant t<t_{n+1}^{(i)}\right) .
$$

These expressions form the basis for the quantification of the disorder average mean-squared displacements, its selfaveraging properties and ergodicity in the following sections.

The key quantity for the determination of the impact of diffusion on disorder sampling in single-disorder realizations and thus the average diffusion behavior is the number of independent replica of $t_{n}$ that can be formed in a single realization,

$$
R_{n}=\frac{(2 n)^{q / 2}}{S_{n}} .
$$

The sampling efficiency $R_{n}$ may be seen as the number of disorder realizations which can be sampled by the noise ensemble in a single-disorder realization. For $q<2$ spatial dimension $R_{n} \sim 1$, which implies that all particles in a single-disorder realization see in average the same disorder. There is no diversification and disorder sampling is minimum. Differences between disorder realizations decrease as the ensemble of particles in single realizations experiences a disorder spectrum that is representative for the ensemble of media. For $q=2$ we find that $R_{n} \sim \ln (n)$. This means that the number of disorder configuration that may be sampled within a single realization increases logarithmically with the number of steps; there is a weak sampling. For $q>2$, we find that $R_{n} \sim n^{q / 2-1}$. As expected, the efficiency of disorder sampling increases with the dimensionality of space. These behaviors have an impact on the self-averaging properties of the mean-square displacement as discussed below. 


\section{Disorder average}

The average diffusion behavior can be described by the disorder average over the particle distribution in Eq. (22),

$$
\bar{p}(\mathbf{x}, t)=\sum_{n=0}^{\infty}\left\langle\delta\left(\mathbf{x}-\mathbf{x}_{n}\right)\right\rangle \frac{1}{R_{n}} \sum_{i=1}^{R_{n}} \overline{\mathbb{I}\left(t_{n}^{(i)} \leqslant t<t_{n+1}^{(i)}\right)} .
$$

Here and in the following the overbar indicates the average over the ensemble of disorder realizations. The disorder ensemble average removes here the dependence of the $t_{n}^{(i)}$ on the singledisorder realizations, such that we can write

$$
\bar{p}(\mathbf{x}, t)=\sum_{n=0}^{\infty}\left\langle\delta\left(\mathbf{x}-\mathbf{x}_{n}\right)\right\rangle p_{n}(t)
$$

where $p_{n}(t)$ is the distribution of numbers of steps needed to reach time $t$,

$$
p_{n}(t)=\overline{\mathbb{I}\left(t_{n} \leqslant t<t_{n+1}\right)} .
$$

The particle time $t_{n}$ after $n$ steps is given by Eq. (16).

In $q>2$ spatial dimensions the number $S_{n}$ of distinct sites visited increases linearly with step number, $S_{n}=b n$, where $b$ depends on the type of lattice on which the medium is discretized, and thus on the geometry of the medium. Here, we consider a simple cubic lattice, for which $b \approx 0.66$ [48]. Accordingly, for $q>2$, the renormalized particle trajectory Eq. (16) can be written as

$$
\mathbf{x}_{N}=\sum_{i=1}^{N} \frac{\ell}{\sqrt{b}} \boldsymbol{\eta}_{i}, \quad t_{N}=\sum_{i=1}^{N} \frac{\tau_{i}}{b},
$$

see Appendix A. This implies that the average particle motion describes a CTRW [1]. For $q \leqslant 2$ it is not possible to formulate the renormalized space-time particle motion Eq. (16) in the form of a CTRW because of the step number dependence of the ratio $S_{n} / n$, which is $S_{n} / n \propto n^{q / 2-1}$ in $q<2$ and $S_{n} / n=$ $1 / \ln (n)$ in $q=1$ spatial dimension.

\section{DIFFUSION BEHAVIOR}

In this section we investigate the diffusion behavior in $q=1,2$, and 3 spatial dimensions both numerically and analytically. We study the disorder ensemble average diffusion behavior as well as diffusion in single-disorder realizations. The media are characterized by a spatially random distribution of retardation coefficients $\theta(\mathbf{x})$ and organized in equisized hypercubes of length $\ell$ as illustrated in Fig. 1. The retardation coefficients $\theta(\mathbf{x})$ are assigned to the hypercubes randomly. This type of medium has a linear tent-shaped correlation function. As discussed in Sec. II A, such media can be considered caricatures of more general random media characterized by a constant correlation distance $\ell$. The spatial random fields $\theta(\mathbf{x})$ generated in this way are stationary and isotropic. The distribution of point values of $\theta(\mathbf{x})$ is denoted by $p_{\theta}(\theta)$. We consider in the following disorder scenarios characterized by

$$
p_{\theta}(\theta) \propto \theta^{-1-\beta},
$$

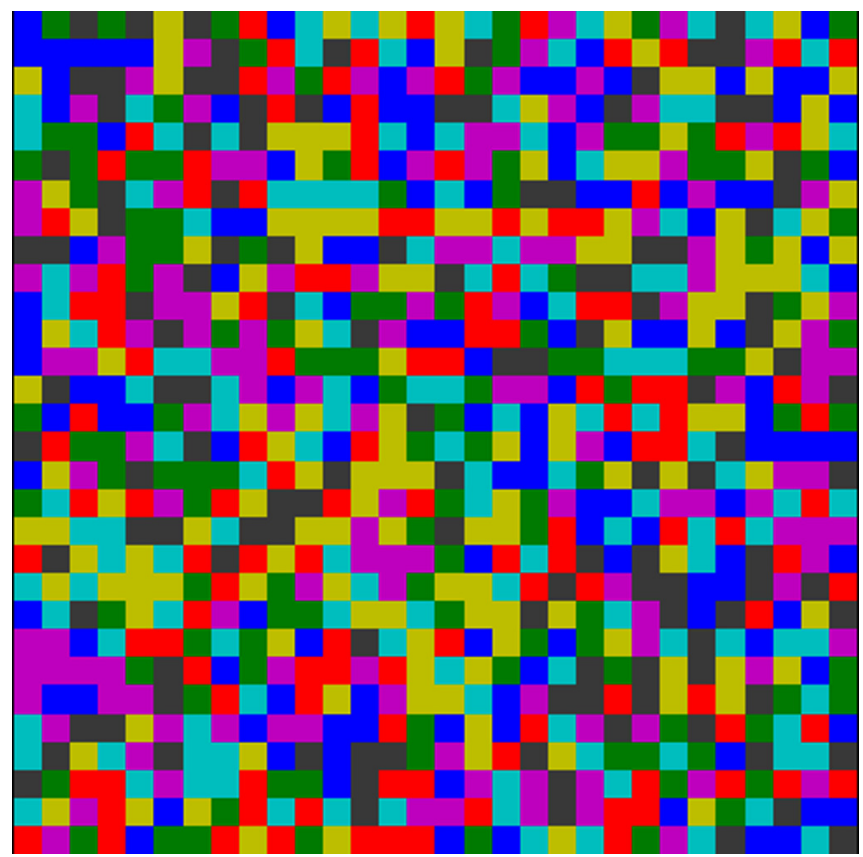

FIG. 1. Realization of a quenched random medium organized in equally sized pixels. The retardation coefficient $\theta(\mathbf{x})$ is distributed according to a heavy-tailed PDF for $\beta=1 / 4$. Different colors denote different values of $\theta(\mathbf{x})$ on a logarithmic scale.

with $\beta>0$ and $\theta \geqslant 1$. According to Eq. (12), this implies a transition time PDF that behaves as

$$
\psi(t) \propto\left(\frac{t}{\tau_{\kappa}}\right)^{-1-\beta} .
$$

Moments of order higher than $\lfloor\beta\rfloor$ are not defined, where $\lfloor\cdot\rfloor$ denotes the floor function. The setup of the numerical random walk simulations and the specific choice for $p_{\theta}(\theta)$ are described in Appendix F. In the following, we first discuss the disorder average behavior, which we then contrast with the diffusion behavior in single-medium realizations.

We study the diffusion behavior in terms of the particle distributions and mean-square displacements. For illustration, we consider the integrated particle distributions $p\left(x_{1}, t\right)=$ $\left\langle\delta\left[x_{1}-x_{1}(t)\right]\right\rangle$ for $q=2$ and 3 . As initial condition we consider an instantaneous particle injection at $\mathbf{x}=\mathbf{0}$. We first consider the disorder average diffusion behavior and briefly summarize the well known long time scaling of the disorder average mean-square displacement $\bar{m}(t)$. We then study the diffusion behavior in single realizations and the sample to sample fluctuations as a function of the spatial dimensions and the disorder distribution.

\section{A. Disorder average behavior}

The disorder average mean-square displacement $\bar{m}(t)=$ $\overline{\left\langle\mathbf{x}(t)^{2}\right\rangle}$ is obtained by averaging Eq. (20) as

$$
\bar{m}(t)=\ell^{2} \overline{n_{t}},
$$

where the overbar indicates the average over the ensemble of realizations. During the first step, $n_{t}$ can be approximated by a Poisson variable whose mean is given by $\bar{n}_{t}=t / \tau_{\kappa} \overline{\theta(\mathbf{0})^{-1}}$. 

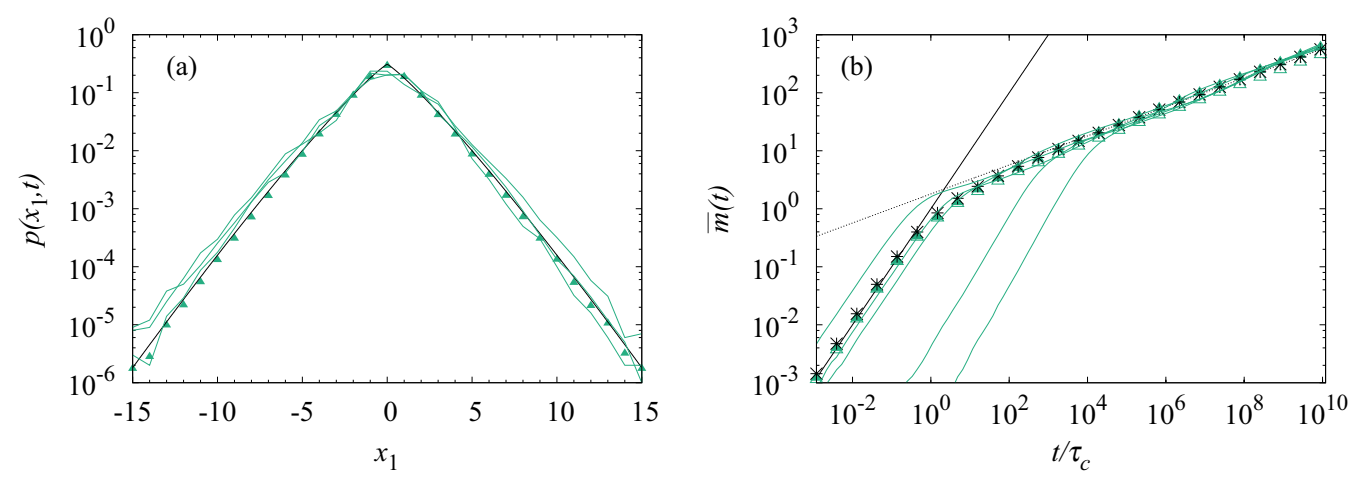

FIG. 2. (a) Concentration profile at $t=20 \tau_{\kappa}$ and (b) disorder average mean-square displacement for a pulse injection into $q=3$-dimensional random media for $\beta=1 / 4$ and $\kappa=1$. The full symbols represent the disorder ensemble average, lines the behaviors in single realizations. (Right) Open symbols denote the behavior of the corresponding annealed disorder model, stars the behavior of the equivalent CTRW discussed in Sec. II C. The solid black line denotes the early-time behavior, Eq. (31); the dashed line denotes the late-time behavior, Eq. (33).

Thus, the early-time behavior of $\bar{m}(t)$ is

$$
\bar{m}(t)=\frac{2 q \kappa}{\theta_{H}} t,
$$

where $\theta_{H}=1 / \overline{\theta^{-1}}$ is the harmonic average of $\theta$. This behavior is valid for times shorter than the characteristic diffusion time $\tau_{c}$ across the injection region of size $\ell$,

$$
\tau_{c}=\theta_{H} \tau_{\kappa}
$$

In the following, we briefly discuss the diffusion behavior for disorder scenarios characterized by infinite and finite mean transition times.

\section{Infinite mean transition time $(0<\beta<1)$}

For infinite mean transition time the disorder average long time diffusion behavior is sub-diffusive. The scalings of the mean-squared displacements for diffusion in quenched disorder depend on the dimensionality of space and are reported in Ref. [1] and derived in the current framework in Appendix D 1.

As discussed in Sec. II C, for $q>2$ dimensions, the disorder average diffusion behavior describes a CTRW and $\bar{m}(t)$ scales as

$$
\bar{m}(t) \propto t^{\beta}
$$

Figure 2(a) shows the areally integrated profile, which behaves as the stretched exponential [39],

$$
\bar{p}\left(x_{1}, t\right) \sim \exp \left[-a\left|x_{1} / \bar{m}(t)\right|^{2 /(2-\beta)}\right],
$$

with $a$ a positive constant and $\bar{m}(t)$ given by Eq. (33). Figure 2(b) shows the numerically obtained $\bar{m}(t)$, the exact early-time behavior, Eq. (31), and the long time scaling, Eq. (33). The behavior of the equivalent renormalized CTRW discussed in Sec. II C and the diffusion behavior in an annealed disorder scenario characterized by $\psi(t)$ are also displayed in Fig. 2(b). Note that the annealed scenario does not account for the renormalization of the space and time increments.

The mean-square displacement in the annealed scenario is equal to the quenched case at short times. It underestimates $\bar{m}(t)$ at times $t>\tau_{c}$ because it does not account for the renormalized time and space increments. Conversely, the equivalent CTRW coincides with the quenched behavior for $t \gg \tau_{c}$. The disorder average behavior behaves as in an equivalent annealed disorder. The quenched nature of the spatial disorder is only revealed in the sample to sample fluctuations as discussed in the next section.

In $q=2$ and $q<2$ dimensions, $\bar{m}(t)$ behaves as

$$
\bar{m}(t) \propto t^{\beta} \ln (t)^{1-\beta}, \quad \bar{m}(t) \propto t^{\frac{2 \beta}{2 \beta-q \beta+q}},
$$

respectively. The mean-square behavior is subdiffusive, but increases faster than in $q>2$. In the following, we focus on $q=1$ and $q=2$ as well as $q>2$ spatial dimensions.

Figures 3(a) and 3(c) show the disorder average particle distributions in $q=2$ and $q=1$ dimensions and illustrate that $\bar{m}(t)$ increases faster as dimension decreases. The profile for $q=2$ dimensions adjusts to the same stretched exponential Eq. (34) as in $q=3$ with $\bar{m}(t)$ given by Eq. (35). For $q=1$ the profile is well described by the stretched exponential [42]

$$
p\left(x_{1}, t\right) \sim \exp \left(-b|x / \bar{m}(t)|^{1+\beta}\right)
$$

with $b$ a positive constant and $\bar{m}(t)$ the disorder average meansquare displacement Eq. (35) for $q=1$.

Figures 3(b) and 3(d) display the disorder average meansquare displacement for $q=2$ and $q=1$ dimensions. For comparison, we plot the diffusion behavior for an annealed disorder scenario characterized by the same $\psi(t)$. The shorttime behavior is given exactly by Eq. (31); the long-time behavior for $t \gg \tau_{c}$ is given by Eq. (35). At short times $t<\tau_{c}$, the annealed and quenched scenarios behave in the same way because the behavior is given by the harmonic average of the residence times in the injection region. At longer times, when particles start sampling the disorder, the space dependency of transition times implies correlation of successive time increments. Thus, in quenched disorder particles visit in average a lower number of different sites than in annealed disorder. Consequently particles encounter a lower number of high retardation sites that determines long-time behavior. As a consequence, mean-square displacement increases slower in annealed than in quenched disorder. 

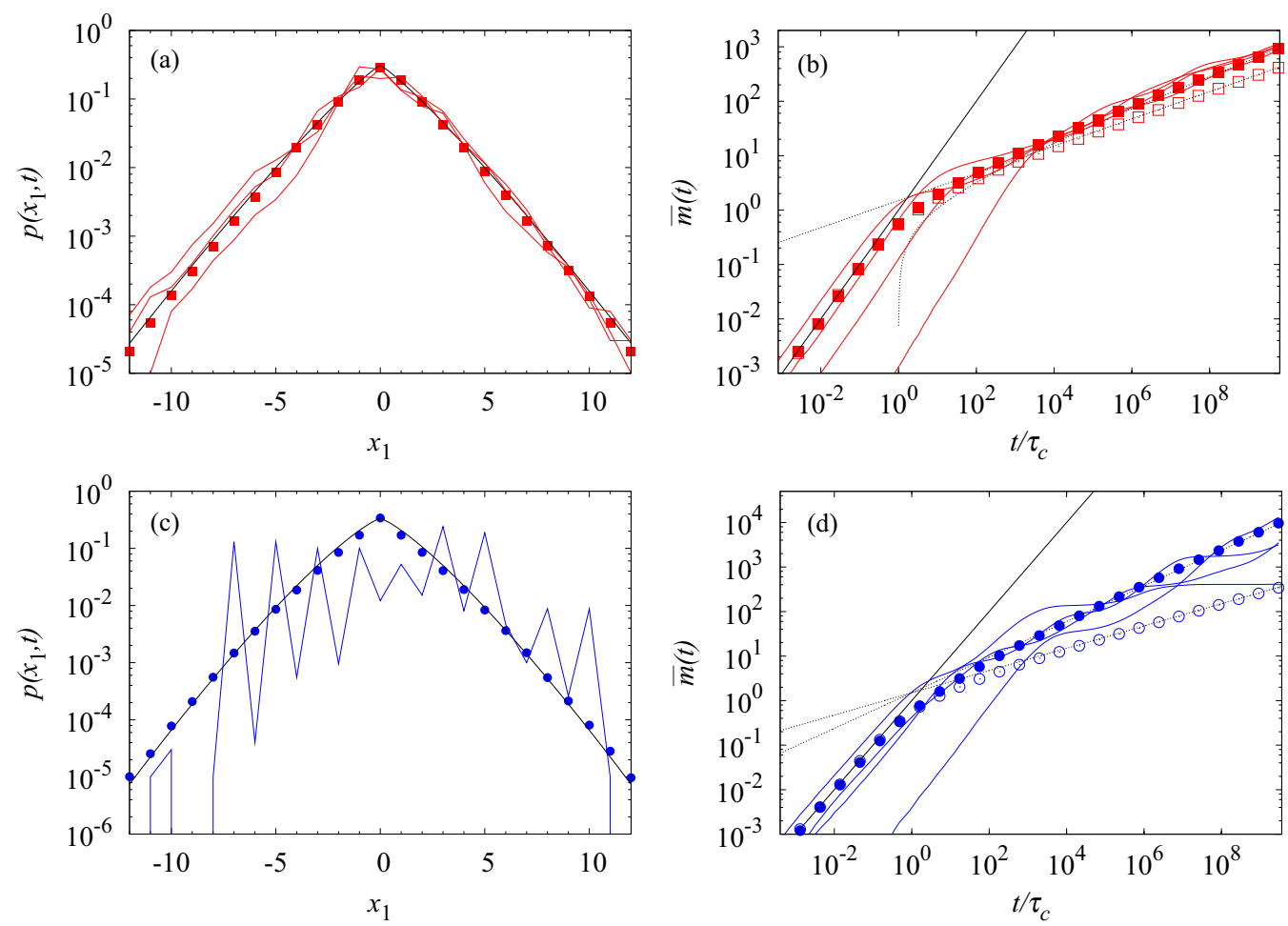

FIG. 3. (a, c) Concentration profiles at $t=20 \tau_{\kappa}$ for a pulse injection into (a) $q=2$ - and (c) $q=1$-dimensional random media for $\beta=1 / 4$ and $\kappa=1$. The full symbols represent the disorder ensemble average, lines the behaviors in single realizations. (b, d) Disorder average mean-square displacement for a pulse injection into (b) $q=2$ - and (d) $q=1$-dimensional random media for $\beta=1 / 4$ and $\kappa=1$. The full symbols represent the disorder average, lines the behaviors in single realizations. Open symbols denote the behavior of the corresponding annealed disorder model. The solid black line denotes the early-time behavior, Eq. (31); the dashed lines denotes the late-time behaviors, Eq. (35).

\section{Finite mean transition time $(\beta>1)$}

Here, we consider media characterized by a power law distribution of retardation coefficients with $\beta>1$. In this case, the mean transition time $\bar{\tau}$ exists. The long-time diffusion behavior is normal. The disorder average mean-square displacement behaves in leading order as (see Appendix D 2)

$$
\bar{m}(t)=\frac{2 q \kappa}{\theta_{A}} t
$$

where $\theta_{A}=\overline{\theta(\mathbf{x})}$ is the arithmetic average of $\theta(\mathbf{x})$. This behavior holds in any dimension. The quenched nature of the disorder manifests in the self-averaging behavior of the mean-square displacement as discussed below.

Figure 4(a) illustrates the particle distribution for $q=1$ and $\beta=3 / 2$. It is well described by a Gaussian distribution. Figure 4(b) shows the disorder average mean-square displacement obtained for $q=1,2$ and 3 dimensions with $\beta=3 / 2$. We observe that $\bar{m}(t)$ scales linearly with time for any dimension. For $t<\tau_{c}$ it behaves as predicted by Eq. (31) and for $t>\tau_{c}$ as Eq. (37).

\section{B. Single-medium realizations}

In this section we study diffusion in single-disorder realizations. We discuss the sample to sample fluctuations of the mean-square displacement and the particle distribution between realizations. The early time mean-square displacement in a single realization is given by

$$
m(t)=\frac{2 q \kappa}{\theta(\mathbf{0})} t .
$$

This behavior persists as long as the particle displacement is smaller than the characteristic length scale $\ell$, this means for times $t<\theta(\mathbf{0}) \tau_{\kappa}$. This time scale is determined by the disorder at the injection point, which varies from realization to realization.

\section{Infinite mean transition time $(0<\beta<1)$}

Figure 3(c) compares the particle distributions at $t=20 \tau_{\kappa}$ after a pulse injection in a single $q=1$-dimensional disorder realizations with the disorder ensemble average. The spatial particle distribution is characterized by multiple peaks which are due to the retention of particles in regions of high $\theta$. Figure 3(d) shows the mean-square displacement for different $q=1$-dimensional disorder realizations. As predicted, at short times the mean-square displacement increases in each realization linearly in time until $\theta(\mathbf{0}) \tau_{\kappa}$ with $\theta(\mathbf{0})$ the retention of the first pixel. At times longer than $\theta(\mathbf{0}) \tau_{\kappa}$, the injected particles start sampling the medium heterogeneity of high and low $\theta$ values. Thus, diffusion at long times is slowed down due to particle retention in regions of high $\theta$ and the mean-square displacement fluctuates around the ensemble mean behavior given by Eq. (35). 

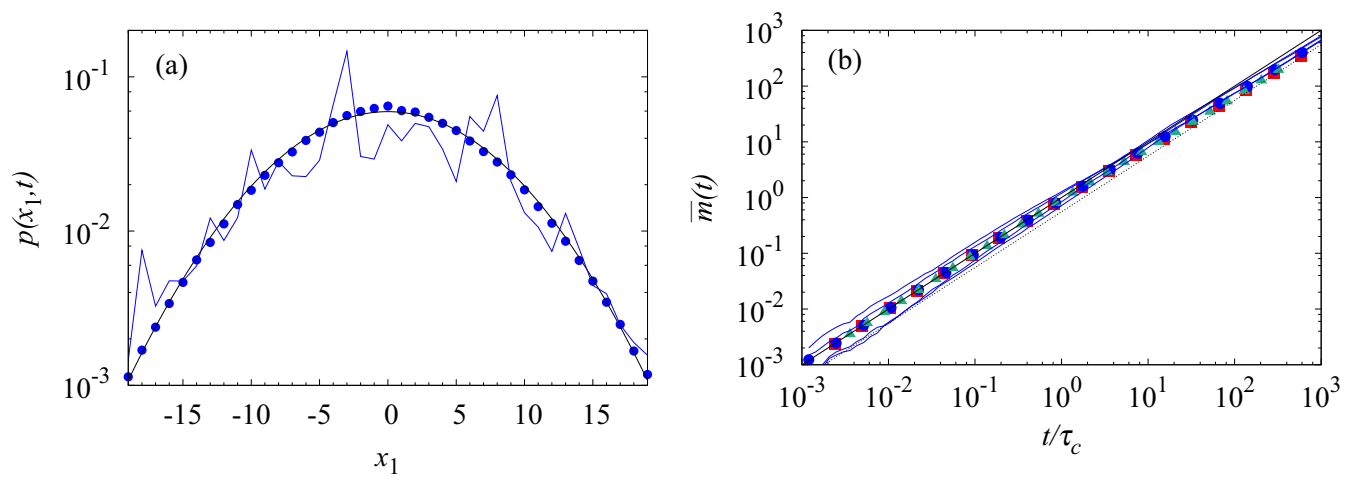

FIG. 4. (a) Concentration profile at $t=20 \tau_{c}$ for a pulse injection into a $q=1$-dimensional random media for $\beta=3 / 2$ and $\kappa=1$. The full symbols represent the disorder average, the line the behavior in a single-medium realization. (b) Disorder average mean-square displacement for a point injection into (triangles) $q=3-$, (squares) $q=2-$, and (circles) $q=1$-dimensional random media. The full symbols represent the ensemble average, the lines the behavior in single $q=1$-dimensional medium realizations. The solid black line denotes the early-time behavior, Eq. (31); the dashed line denotes the late-time behavior, Eq. (37).

Figure 5 shows the particle distribution in a single realization of a $q=2$-dimensional random medium. We observe similar localization features as in $q=1$. The corresponding integrated particle distributions are compared in Fig. 3(a) to the average distribution. The integrated profiles are naturally much smoother than for $q=1$. The mean-square displacement displayed in Fig. 3(b) shows the exact behavior given by Eq. (38) for $t<\theta(\mathbf{0}) \tau_{\kappa}$. As in $q=1, m(t)$ slows down and fluctuates around the ensemble mean given by (35). The fluctuations are smaller than in $q=1$ due to the more efficient disorder sampling. This feature is even more pronounced in $q=3$.

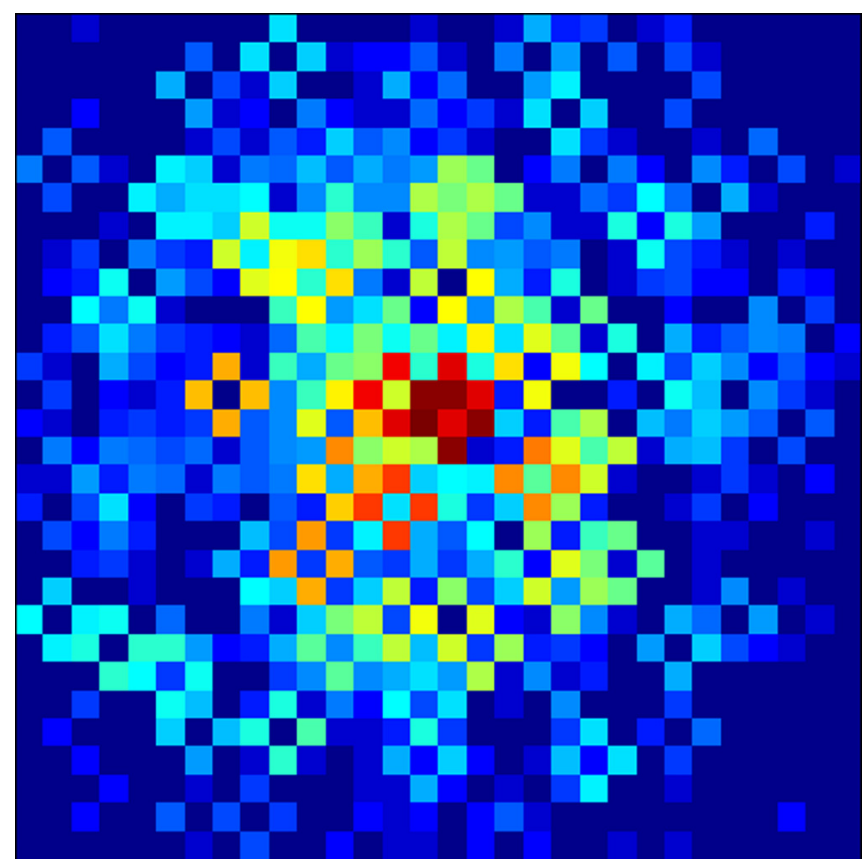

FIG. 5. Particle distribution for a pulse injection into a $q=2$ dimensional heterogeneous medium for $\beta=1 / 4$ and $\kappa=1$. Different colors denote different values of concentration with a logarithmic color scheme.
Figure 6 shows the particle distribution in a section of a $q=3$-dimensional medium. Again, we observe certain localization features, but the particle distribution is smoother than in $q=2$ due to more efficient disorder sampling. The more efficient disorder sampling is also manifest in the behavior of the mean-square displacement shown in Fig. 2(b). While the short-time behavior depends strongly on the single-medium properties, the long-time behavior fluctuates much less about the mean behavior given by (33) than in $q<3$.

In summary, fluctuations of the particle distribution and the mean square displacement are decreasing with increasing spatial dimension. This is due to the increase in the sampling efficiency with $q$. As discussed in Sec. II B, the number $R_{n}$ of

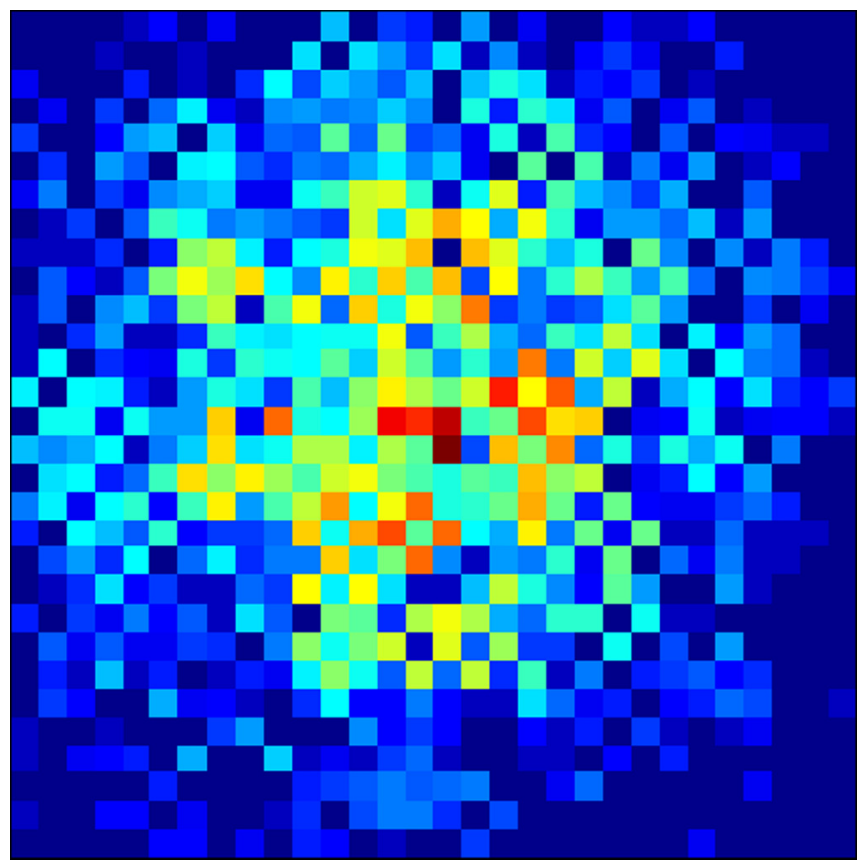

FIG. 6. Particle distribution across a section for a pulse injection into a $q=3$-dimensional heterogeneous medium for $\beta=1 / 4$ and $\kappa=1$. Different colors denote different values of concentration with a logarithmic color scheme. 
possible sites that may be explored evolves with step number as $R_{n} \propto n^{q / 2-1}$ and the ability $S_{n}$ of sampling new sites increases as a function of $q$.

\section{Finite mean transition time $(\beta>1)$}

Here we consider media characterized by the disorder distribution given by Eq. (28) with $\beta>1$, which implies that the ensemble mean transition time $\bar{\tau}$ is finite. Figure 4(b) illustrates the mean-square displacements in single $q=1$-dimensional disorder realizations with $\beta=3 / 2$. For compactness, we omit the behaviors for $q=2$ and $q=3$. At short times, for $t<\theta(0) \tau_{\kappa}, m(t)$ increases linearly with time as in Eq. (38). At long times, the behaviors are approximately linear in all realizations and fluctuate around the disorder average given by (37). For $q=2$ and $q=3$, the behaviors are similar, but the fluctuations around the mean behavior decrease with increasing spatial dimension (not shown).

\section{SELF-AVERAGING}

The differences in the diffusion behaviors between disorder realizations depend on the efficiency by which the diffusing particles sample the heterogeneity in single disorder realizations. This efficiency is expressed by $S_{n}$, the average number of distinct sites visited after $n$ random walk steps, and by the number of different disorder configurations particles are able to see in a single realization, which is measured by $R_{n}$ defined by Eq. (23). As discussed in Sec. II B, in $q=1$ dimension the number of different disorder configurations accessible to the diffusing particles within a single realization is of the order of 1 . Thus, we expect that sample to sample fluctuations persist, while in $q \geqslant 2, R_{n}$ increases with step number. In the following, we discuss how these attributes impact on the sample to sample fluctuations of the meansquare displacement for anomalous as well as normal average transport.

The fluctuations of the mean-square displacement $m(t)$ are quantified by its disorder variance,

$$
\sigma_{m}^{2}(t)=\overline{m(t)^{2}}-\bar{m}(t)^{2},
$$

where $m(t)$ for a single realization is given by Eq. (20). Again, we obtain an exact result for early times $t<\tau_{\kappa} / \theta_{H}$. Using Eq. (38) for $m(t)$ and Eq. (31) for its disorder average in Eq. (39), we obtain

$$
\sigma_{m}^{2}(t)=4 q \kappa^{2} t^{2} \overline{\left(\theta^{-1}-\theta_{H}^{-1}\right)^{2}} .
$$

For times $t \gg \tau_{c}$, we derive in Appendix B the following compact expression:

$$
\sigma_{m}^{2}(t)=\ell^{4} \overline{n_{t}\left(n_{t}-\bar{n}_{t}\right) / R_{n_{t}}}
$$

The mean-square displacement is quantified in terms of the disorder averages of the renewal process $n_{t}=\max \left(n \mid t_{n} \leqslant t\right)$, which describes the number of steps needed to reach time $t$ in the process $t_{n}$ defined in Eq. (16). The self-averaging property is quantified by the relative variance $[1,49,50]$,

$$
\Sigma(t)=\frac{\sigma_{m}^{2}(t)}{\bar{m}(t)^{2}} .
$$

If $\lim _{t \rightarrow \infty} \Sigma(t)=0$, diffusion is referred to as self-averaging, if $\lim _{t \rightarrow \infty} \Sigma(t)>0$ as non-self-averaging. Note that the fact that $\Sigma(t)$ goes to 0 with increasing time does not imply that the variance disappears. It may, in fact, be large. However, the ratio of fluctuation to mean value goes to 0 , which means that the relative error of the disorder ensemble averaged quantity disappears in the limit of infinite times. This property is defined here as self-averaging in agreement with Aslangul et al. [49] and Bouchaud and Georges [1], who consider the evolution of the relative variance over time, and Wiseman and Domani [50], who consider the evolution of the relative variance as a function of the spatial size of a disorder sample.

In the following, we discuss the variance of the mean square displacement $m(t)$ and its self-averaging behavior for disorder scenarios that are characterized by infinite and finite mean transition times. The detailed calculations leading to the results discussed in the following can be found in Appendix E and, for the case of infinite mean transition time in Ref. [41], which we summarize here for completeness.

\section{A. Infinite mean transition time $(0<\beta<1)$}

The disorder average mean-square displacement for $0<$ $\beta<1$ is subdiffusive in any spatial dimension. The variance of $m(t)$ in $q=1$ spatial dimensions behaves for $t \gg \tau_{c}$ as

$$
\sigma_{m}^{2}(t) \propto t^{\frac{4 \beta}{2 \beta-q \beta+q}} .
$$

Thus, using Eqs. (43) and (35) for the relative variance we have $\Sigma(t) \sim 1$. This implies that the mean-square displacement is not self-averaging for $q=1$ as expected from the constant number of possible realities $\left(R_{n} \sim 1\right)$ that may be sampled in a single realization. Nevertheless, Eq. (43) implies that $m(t) \propto$ $\bar{m}(t) \propto t^{\frac{2 \beta}{2 \beta-\alpha \beta+q}}$, as discussed in the previous section.

For $q=2$, one finds the following long-time scaling:

$$
\sigma_{m}^{2}(t) \propto t^{2 \beta} \ln (t)^{1-2 \beta} .
$$

Here the relative variance $\Sigma(t) \propto 1 / \ln (t)$; see Eq. (35). The process is self-averaging, but the relative variance decays very slowly. This behavior is in line with the slow increase of $S_{n} \sim$ $\ln (n)$ of new sites that may be sampled.

For $q>2$, the disorder variance of $m(t)$ scales as

$$
\sigma_{m}^{2}(t) \propto t^{\beta(3-q / 2)} .
$$

This implies, together with Eq. (33), that the relative variance scales as $\Sigma(t) \propto t^{\beta(1-q / 2)}$. The diffusion behavior is selfaveraging and the decay of the relative variance decreases with the spatial dimension. Again, this behavior is reflected by the increasing sampling efficiency expressed both by the ability of sampling new sites, which scales as $S_{n} \sim n$ and the number of different realities that may be sampled by the random walk, which scales as $R_{n} \sim n^{q / 2-1}$.

Figure 7(a) shows the temporal evolution of $\sigma_{m}^{2}(t)$ sampled from numerical Monte Carlo simulations using $10^{4}$ realizations of the random medium for $\beta=1 / 4$ and $q=1,2$, and 3. The analytical results in Eq. (40) for early times and the late time scalings given in (43) and (44) are consistent with the numerical simulation data. At short times $t \ll \tau_{c}$ there is a quadratic increase with time of uncertainty of the mean-square displacement, which is due to the poor disorder sampling; 

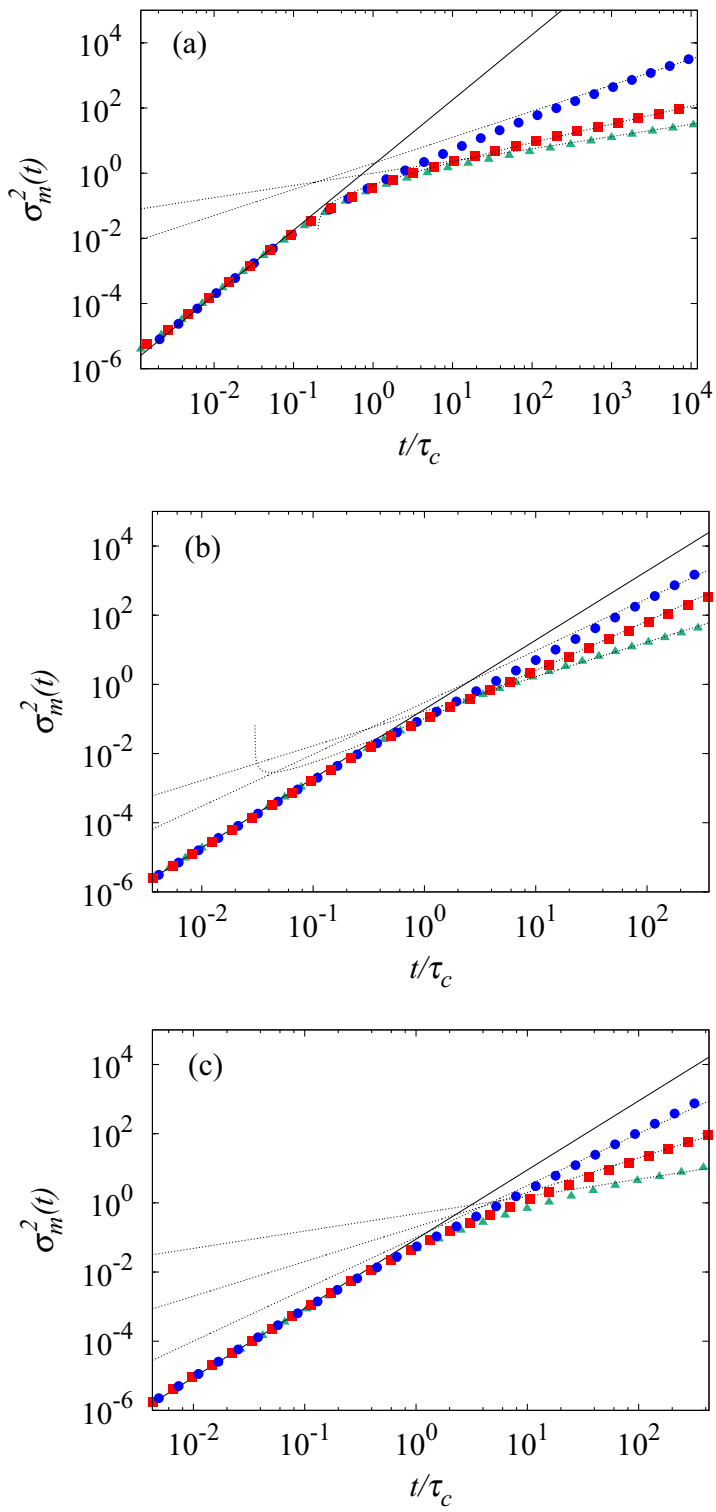

FIG. 7. Evolution of the variance $\sigma_{m}^{2}(t)$ for a pulse injection of $10^{5}$ particles into $10^{3}$ realizations of (triangles) $q=3$, (squares) $q=2$, and (circles) $q=1$-dimensional random media with $\kappa=1$ and (a) $\beta=1 / 4$, (b) $\beta=3 / 2$, and (c) $\beta=5 / 2$. The solid black line shows the analytical early-time behavior Eq. (40), the dashed black lines the late-time behaviors (top) Eqs. (43)-(45), (middle) Eqs. (46) and (47), and (bottom) Eq. (49).

particles have not yet diffused over lengths larger than the characteristics disorder scale $\ell$. For times $t>\tau_{c}$, particles start seeing the disorder and the variance increase slows down.

\section{B. Finite mean transition time $(\beta>1)$}

For finite mean transition time, the disorder average meansquare displacement evolves linearly with time. This means that average particle motion is diffusive, i.e., $m(t) \propto t$ for any dimension $q$. The behavior of the variance of $m(t)$, however, depends on the spatial dimension and on the value of $\beta$. We distinguish the cases $1<\beta<2$ for which the mean transition time is finite while $\overline{\tau^{2}}$ is infinite, and the case $\beta>2$ for which both the mean and the mean-square transition time are finite. The results presented in the following are derived in Appendix E.

\section{Self-averaging property for $1<\beta<2$}

For $1<\beta<2$ the variance of the transition time is infinite. The scaling of $\sigma_{m}^{2}(t)$ for $q=1$ is

$$
\sigma_{m}^{2}(t) \propto(t / \bar{\tau})^{\frac{4-q(\beta-1)}{2}} .
$$

This indicates that the variance increases with time faster than in the case $0<\beta<1$. The relative variance, however, decays algebraically with time as $\Sigma(t) \propto(t / \bar{\tau})^{-\frac{q(\beta-1)}{2}}$. The mean-square displacement is self-averaging despite the poor disorder sampling in $q=1$ for which the number of possible disorder realizations that may be sampled is $R_{n} \sim 1$. Disorder sampling is still poor as expressed by the slow decay of the relative variance. For $q=2$, we find

$$
\sigma_{m}^{2}(t) \propto(t / \bar{\tau})^{3-\beta} \ln (t / \bar{\tau})^{\beta-2} .
$$

The relative variance here decreases as $\Sigma(t) \propto$ $(t / \bar{\tau})^{1-\beta} \ln (t / \bar{\tau})^{\beta-2}$.

For $q>2$, we obtain for $\sigma^{2}(t)$ the long-time behavior,

$$
\sigma_{m}^{2}(t) \propto(t / \bar{\tau})^{4-\beta-q / 2} .
$$

Similar to the case $q \leqslant 2$ dimensions, the disorder variance increases faster than in the case of an infinite mean transition time. The relative variance, however, scales as $\Sigma(t) \propto$ $(t / \bar{\tau})^{2-\beta-q / 2}$, which indicates that diffusion is self-averaging. These behaviors are illustrated in Fig. 7(b) for $\beta=3 / 2$ for $q=1,2$, and 3 . At early times, we observe the characteristic quadratic increase, which slows down when the particles start experiencing the disorder.

\section{Self averaging property for $\beta>2$}

For $\beta>2$, both the mean and mean-squared transition times are finite. In this case, one obtains for the disorder variance $\sigma_{m}^{2}(t)$

$$
\sigma_{m}^{2}(t) \propto(t / \bar{\tau})^{2-q / 2} .
$$

This implies that the relative variance behaves as $\Sigma(t) \propto$ $(t / \bar{\tau})^{-q / 2}$. As expected the behavior is always self-averaging, but the relative variance decreases slower with decreasing spatial dimension. Figure 7(c) illustrates the behavior of $\sigma_{m}^{2}(t)$ for $\beta=5 / 2$ and $q=1,2$, and 3 spatial dimensions.

\section{ERGODICITY}

In this section, we study the issue of ergodicity. While self-averaging refers to the sample to sample fluctuations of the mean-square displacement $m(t)$ with respect to its disorder average $\bar{m}(t)$, the ergodicity property considers the fluctuations of the time-averaged mean-square displacement with respect to its noise average. In other words, self-averaging refers to the variability of noise averages between disorder realizations, while ergodicity refers to variability of time averages between noise realizations (particles) in single-disorder realizations.

The mean-square displacement of $\mathbf{x}(s)$ in Eq. (8) is ergodic in operational time $s$ because it describes an ordinary random walk characterized by a stationary increment. This is different 
for the process $\mathbf{x}(t) \equiv \mathbf{x}[s(t)]$ that in clock time samples the heterogeneity slower. Ergodicity is related to the ability of a single particle to sample the available fluctuation spectrum in a finite time. The time process here is directly related to the disorder process as given in Eq. (8). Thus, disorder sampling in time is limited by the disorder itself. To probe the ergodicity of the random walk, we consider the time-averaged particle increments [35],

$$
m_{\Delta}(t)=\frac{1}{t-\Delta} \int_{0}^{t-\Delta} d t^{\prime}\left[\mathbf{x}\left(t^{\prime}+\Delta\right)-\mathbf{x}\left(t^{\prime}\right)\right]^{2} .
$$

It is interesting to note that this quantity is related to the empirical variogram [51], which is used in geostatistics to characterize the variability of a spatial random field. Equation (50) is typically referred to as time-averaged mean-square displacement in the literature. Here, we refer to it as the time average of the increment process $\mathbf{x}_{\Delta}(t) \equiv \mathbf{x}(t+\Delta)-\mathbf{x}(t)$. Using Eq. (8), we can write

$$
\mathbf{x}_{\Delta}(t)=\sqrt{2 \kappa} \boldsymbol{w}_{\Delta}(t), \quad \boldsymbol{w}_{\Delta}(t)=\int_{s(t)}^{s(t+\Delta)} d s^{\prime} \boldsymbol{\eta}\left(s^{\prime}\right),
$$

with $\left\langle\boldsymbol{w}_{\Delta}(t)\right\rangle=\mathbf{0}$ and $\left\langle\boldsymbol{w}_{\Delta}(t)^{2}\right\rangle=q[s(t+\Delta)-s(t)]$. Thus, we may write Eq. (50) in a weak sense as

$$
m_{\Delta}(t)=\frac{2 q \kappa}{t-\Delta} \int_{0}^{t-\Delta} d t^{\prime}\left[s\left(t^{\prime}+\Delta\right)-s\left(t^{\prime}\right)\right] .
$$

We furthermore expand this expression for $t \gg \Delta$ to obtain

$$
m_{\Delta}(t)=\frac{2 q \Delta \kappa}{t} s(t)+\cdots,
$$

where the dots denote contributions of order $\Delta^{2}$. We define now the quantity

$$
m_{a}(t) \equiv \frac{m_{\Delta}(t)}{\Delta} t=2 q \kappa s(t),
$$

which can be seen as the time-averaged mean-square displacement while $m_{\Delta}(t) / 2 \Delta$ denotes an apparent diffusion coefficient. Note that Lubelski et al. [36] suggest to consider $m_{\Delta}(t) / 2 t$ as an effective diffusion coefficient. These authors focus on the displacement increment as a function of the time increment $\Delta$, while here, we study the long-time behavior of the time-averaged mean-square displacement. According to Eq. (5), the noise mean of $m_{a}(t)$ is equal to the mean-square displacement $\left\langle m_{a}(t)\right\rangle=m(t)$. The process $s(t)$ is identified in the coarse grained random walk Eq. (9) by $s(t) \equiv \tau_{\kappa} n_{t}$, such that

$$
m_{a}(t)=\ell^{2} n_{t} .
$$

The disorder average of $m_{a}(t)$ is equal to $\bar{m}(t)$. Note that Miyaguchi and Akimoto [38] defined a diffusion coefficient as $D_{t}=n_{t} / t$, which is related to $m_{a}(t)$ as $D_{t}=m_{a}(t) / \ell^{2} t$. These authors study the full distribution of $D_{t}$ over the disorder ensemble as well as its disorder mean and variance. The disorder mean of $D_{t}$ is related to the disorder mean-square displacement $\bar{m}(t)$ as $\bar{D}_{t}=\bar{m}(t) / \ell^{2} t$.

Ergodicity of the mean-square displacement is measured in terms of the variance of $m_{a}(t)$ with respect to its noise average $m(t)$,

$$
\left.\sigma_{a}^{2}(t)=\left\langle\left[m_{a}(t)-m(t)\right\rangle\right]^{2}\right\rangle=\ell^{4}\left(\left\langle n_{t}^{2}\right\rangle-\left\langle n_{t}\right\rangle^{2}\right) .
$$

Let us first consider the short-time behavior of $\sigma_{a}^{2}(t)$. The variance of $m_{a}(t)$ in a single realization is given by

$$
\sigma_{a}^{2}(t)=\frac{\ell^{4} t}{\tau_{\kappa} \theta(\mathbf{0})}=\ell^{2} m(t),
$$

because at the first step in a single-medium realization, the transition time is exponentially distributed with mean $\theta(\mathbf{0}) \tau_{\kappa}$. This renders $n_{t}$ approximately a Poisson variable as long as $\left\langle n_{t}\right\rangle \leqslant 1$. Thus, in the diffusive short-time regime, $\sigma_{a}^{2}(t)$ behaves as the mean-square displacement, see Eq. (38). Its disorder average is given at short times $t<\tau_{\kappa}$ by

$$
\bar{\sigma}_{a}^{2}(t)=\ell^{2} \frac{2 q \kappa t}{\theta_{H}} .
$$

To quantify this variance in a systematic way at long times $t \gg \tau_{c}$, we consider the disorder average of Eq. (56), which can be tautologically rewritten as

$$
\bar{\sigma}_{a}^{2}(t)=\ell^{4}\left(\overline{\left\langle n_{t}^{2}\right\rangle}-\overline{\left\langle n_{t}\right\rangle^{2}}\right)-\ell^{4}\left(\overline{\left\langle n_{t}\right\rangle^{2}}-{\overline{\left\langle n_{t}\right\rangle}}^{2}\right) .
$$

The second term on the right is identified with the variance $\sigma_{m}^{2}(t)$ defined by Eq. (39). The first term on the right is the disorder variance of the step number process $n_{t}$, which is independent of the noise in a single-disorder realization,

$$
\sigma_{n}^{2}(t)=\overline{n_{t}^{2}}-{\overline{n_{t}}}^{2} \text {. }
$$

Thus, $\bar{\sigma}_{a}^{2}(t)$ is given by

$$
\bar{\sigma}_{a}^{2}(t)=\ell^{4} \sigma_{n}^{2}(t)-\sigma_{m}^{2}(t) .
$$

This implies that $\sigma_{n}^{2}(t) \geqslant \sigma_{m}^{2}(t) / \ell^{4}$ due to the positiveness of the variance. Thus, the long-time behavior of $\bar{\sigma}_{a}^{2}(t)$ is dominated by $\sigma_{n}^{2}(t)$. The ratio of $\sigma_{n}^{2}(t)$ and $\bar{n}_{t}^{2}$ has been termed ergodicity-breaking parameter EB in the literature [52],

$$
\mathrm{EB}=\frac{\sigma_{n}^{2}(t)}{\bar{n}_{t}^{2}} .
$$

If the value of $\mathrm{EB}$ is nonzero in the limit of infinite times, one speaks of weak ergodicity breaking. Note that $\sigma_{n}^{2}(t)=\left[\overline{m_{a}(t)^{2}}-\bar{m}(t)^{2}\right] / \ell^{4}$, this means, it is proportional to the disorder variance of the time-averaged mean-square displacement. Note also that EB is equal to the relative disorder variance of the diffusion coefficient $D_{t}$ studied by Miyaguchi and Akimoto [38]. Specifically, the variance $\sigma_{n}^{2}(t)$ is related to the disorder variance of $D_{t}$ as $\sigma_{n}^{2}(t)=\left(\overline{D_{t}^{2}}-\bar{D}_{t}^{2}\right) t^{2}$.

Appendix $C$ derives the asymptotic long-time behaviors for the moments and the PDF of $n_{t}$ with respect to the disorder average for $q$ dimensions and for disorder scenarios that are characterized by infinite and finite mean transition times. In the following, we discuss $m_{a}(t)$ and its disorder variance in terms of $\sigma_{n}^{2}(t)$ for different values of $q$ and $\beta$.

\section{A. Infinite mean transition time $(0<\beta<1)$}

As outlined above, for infinite mean transition time, $m(t)$ evolves subdiffusively with time in any spatial dimension. The time-averaged mean-square displacement $m_{a}(t)$ shows subdiffusive scaling as illustrated in Fig. 8 with significant fluctuations around the noise mean $m(t)$. As outlined above, these fluctuations can be quantified in terms of the disorder 


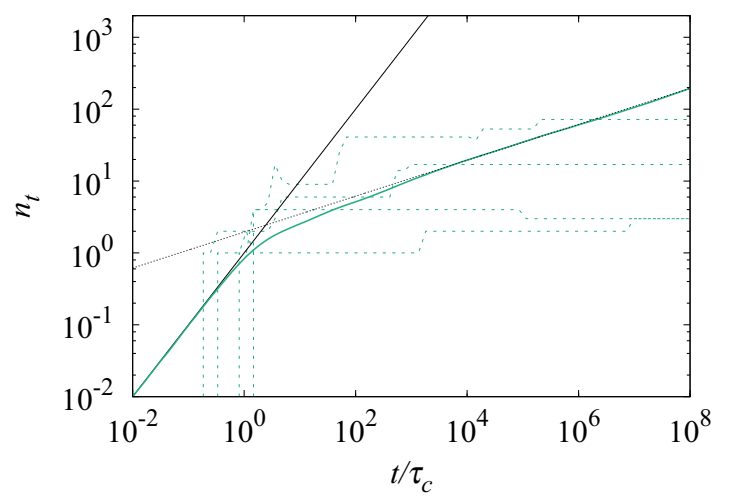

FIG. 8. Evolution of the time-averaged mean-square displacement $m_{a}(t)$ defined by Eq. (55) (dashed) for four particle trajectories, and the noise average $m(t)$ for (solid) $10^{5}$ particles injected at the origin of a $q=3$ heterogeneous medium for $\beta=1 / 4$. The solid black line shows the analytical early-time behavior, Eq. (38); the dashed black lines the late-time behavior, Eq. (33).

variance $\sigma_{n}^{2}(t)$. Miyaguchi and Akimoto [38] report results for the variance of $D_{t}$, which is equivalent to $\sigma_{n}^{2}(t)$. Also, these authors report explicit expressions for EB. In the following, we summarize these results for completeness before we discuss the behavior of $\sigma_{n}^{2}(t)$ and thus $\bar{\sigma}_{a}^{2}(t)$ for disorder distributions characterized by finite mean transition times. by

For $q=1$, the scaling of the disorder variance $\sigma_{n}^{2}(t)$ is given

$$
\sigma_{n}^{2}(t) \propto t^{\frac{4 \beta}{2 \beta-q \beta+q}}
$$

see also Eq. (D6). It scales in the same way as the disorder variance of $m(t)$ given in Eq. (43). As $\bar{n}_{t} \propto t^{\frac{2 \beta}{2 \beta-q \beta+q}}$, the ergodicity breaking parameter is $\mathrm{EB} \sim 1$. This means that diffusion is here weakly ergodicity breaking.

For $q=2$ dimensions, $\sigma_{n}^{2}(t)$ behaves as

$$
\sigma_{n}^{2}(t) \propto t^{2 \beta} \ln (t)^{2-2 \beta},
$$

see also Eq. (D9). This implies that the disorder variance of $\bar{\sigma}_{a}^{2}(t)$ increases faster than $\sigma_{m}^{2}(t)$ given by Eq. (44). In fact, $\sigma_{n}^{2}(t)$ scales as $\bar{n}_{t}^{2}$. Thus, the ergodicity breaking parameter EB $\sim 1$ and again, diffusion is weakly ergodicity breaking.

For $q>2$, the scaling for the disorder variance of $n_{t}$ is

$$
\sigma_{n}^{2}(t) \propto t^{2 \beta}
$$

see also Eq. (D11). The variance $\sigma_{n}^{2}(t)$ scales in the same way as $\bar{n}_{t}^{2}$ and thus, again EB $\sim 1$. Also in $q=3$, diffusion is weakly ergodicity breaking. These behaviors are illustrated in Fig. 9 and compared with data from numerical random particle tracking simulations. Explicit expressions for EB can be found in Miyaguchi and Akimoto [38].

\section{B. Finite mean transition time $(\boldsymbol{\beta}>1)$}

For disorder scenarios that are characterized by finite mean transition times $\bar{\tau}<\infty$, the average diffusion behavior is normal and the diffusion is asymptotically self-averaging. The time-averaged mean-square displacement $m_{a}(t)$ evolves

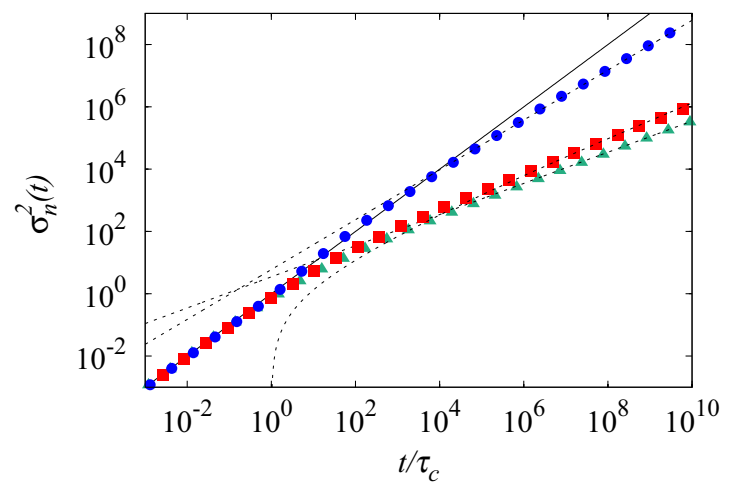

FIG. 9. Evolution of the variance $\sigma_{n}^{2}(t)$ for a pulse injection into $10^{3}$ realizations of a (circles) $q=1$, (squares) $q=2$, and (triangles) $q=3$ random medium for $\beta=1 / 4$. The solid black line shows the analytical early-time behavior, Eq. (58); the dashed black lines the late-time behaviors, Eqs. (63)-(65).

approximately linear but fluctuates about the noise mean $m(t)$ as illustrated in Fig. 10.

For $q=1$, we obtain from Eq. (D14) for $\sigma_{n}^{2}(t)$ the following scaling behavior:

$$
\sigma_{n}^{2}(t) \propto(t /\langle\tau\rangle)^{2+\frac{q(1-\beta)}{2}} .
$$

The behavior for $\beta>2$ is obtained by setting $\beta=2$. Since the disorder mean $\bar{n}_{t}$ scales as $t$, the ergodicity breaking parameter behaves as $\mathrm{EB} \propto(t /\langle\tau\rangle)^{\frac{q(1-\beta)}{2}}$. This means it decreases relatively slowly with time as $t^{-\gamma}$, where $0<\gamma \leqslant q / 2$.

For $q=2$, Eq. (D17) gives

$$
\sigma_{n}^{2}(t) \propto(t / \bar{\tau})^{3-\beta} \ln (t / \bar{\tau})^{\beta-1}
$$

Again, the behavior for $\beta>2$ is obtained by setting $\beta=$ 2 . The ergodicity breaking parameter here scales as EB $\propto$ $[(t / \bar{\tau}) / \ln (t / \bar{\tau})]^{1-\beta}$. Thus, it decays as $[t / \ln (t)]^{-\gamma}$ with $0<$ $\gamma \leqslant 1$.

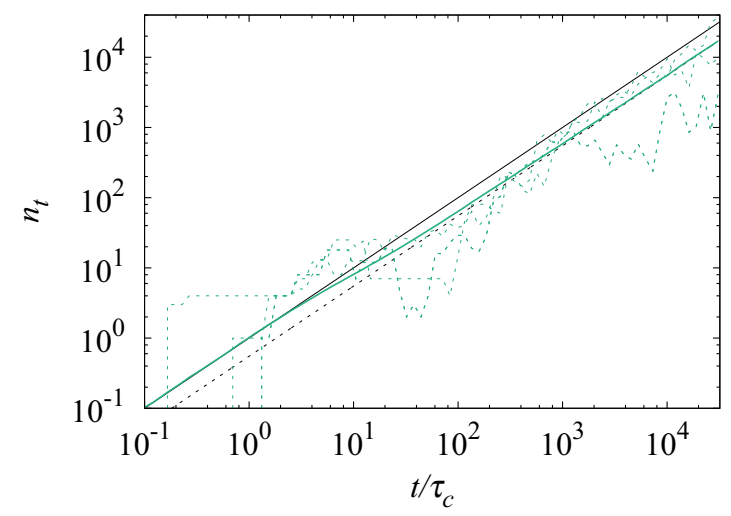

FIG. 10. Evolution of the time-averaged mean-square displacement $m_{a}(t)$ defined by Eq. (55) (dashed) for four particle trajectories, and the noise average $m(t)$ for (solid) $10^{5}$ particles injected at the origin of a $q=3$ heterogeneous medium for $\beta=3 / 2$. The solid black line shows the analytical early-time behavior, Eq. (38); the dashed black lines the late-time behavior, Eq. (37). 


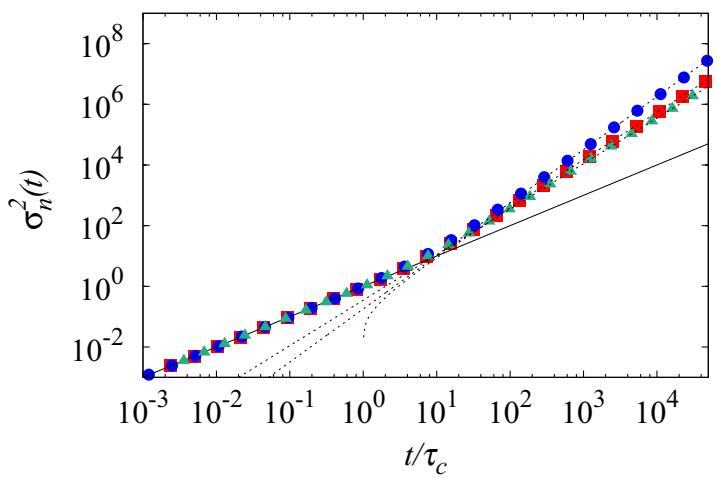

FIG. 11. Evolution of the variance $\sigma_{n}^{2}(t)$ for a pulse injection into $10^{3}$ realizations of a (circles) $q=1$, (squares) $q=2$, and (triangles) $q=3$ random medium for $\beta=3 / 2$. The solid black line shows the analytical early-time behavior, Eq. (58); the dashed black lines the late-time behaviors, Eqs. (66)-(68).

For $q>2$, we obtain from Eq. (D20),

$$
\sigma_{n}^{2}(t) \propto(t /\langle\tau\rangle)^{3-\beta}
$$

The ergodicity breaking parameter scales as EB $\propto(t / \bar{\tau})^{1-\beta}$. It decays as $t^{-\gamma}$ with $0<\gamma \leqslant 1$. Thus, while the timeaveraged mean-square displacement is asymptotically ergodic, its variance decays algebraically with time. Figure 11 shows the evolution of the disorder variance of $n_{t}$ in $q=1,2$, and 3 dimensions from numerical random walk simulations.

For disorder scenarios characterized by a finite mean transition time, diffusion is asymptotically ergodic and selfaveraging, as discussed in Sec. IV. Thus, the ensemble of particles is representative of individual particle trajectories (ergodicity), and the disorder ensemble is representative of the diffusion behavior in single realization (self-averaging).

\section{SUMMARY AND CONCLUSIONS}

We study self-averaging and ergodicity of diffusion in heterogeneous media. Ergodicity refers to the question whether the particle ensemble statistics in a single realization are representative of the temporal statistics sampled along a single-particle trajectory. It is a fundamental property for the inference of stochastic particle rules from single-particle tracking observations. The notion of ergodicity is different from the the notion of self-averaging. The latter refers to the representation of the particle statistics in a single-medium realization through the disorder ensemble statistics. It is a fundamental condition for the use of stochastic modeling for the prediction of diffusion behavior in heterogeneous media. If these properties apply, they are approached asymptotically.

We analyze these behaviors for diffusion in heterogeneous media that are characterized by spatially variable retardation coefficients, which quantifies particle retention due to physical and chemical interactions with the host medium. The retardation coefficient is modeled as a stationary spatial random field. The diffusion problem is coarse-grained on the characteristic heterogeneity length scale and numerically implemented using a TDRW. We derive an explicit expression for the noise average in a single realization, which depends on the number $R_{n}$ of different disorder realizations that the noise ensemble may sample after $n$ diffusion steps in a single-disorder realization. As is well known, for $q>2$, the average particle motion in this model describes a CTRW. We derive the renormalized space and time increments to match the long time diffusion behavior obtained from the TDRW simulations for $q=3$.

We then focus on the mean-square particle displacement and particle distributions averaged over the disorder ensemble and in single-disorder realizations. We derive explicit expressions for the early-time behavior of the mean-square displacement and confirm through the numerical simulations the known analytical results for the asymptotic scaling of the mean-square displacement and disorder average particle distribution for $q \geqslant 1$ spatial dimensions. The disorder ensemble average behavior is contrasted to the evolution of the mean-square displacement and particle distributions in single realizations. We observe significant sample to sample fluctuations and segregation of the particle distribution due to localized strong retention, which are more significant for decreasing spatial dimension. These observations are discussed in the light of the sampling efficiency $R_{n}$, which depends on the spatial dimension $q ; R_{n}$ increases faster with $n$ for increasing $q$. For $q=1, R_{n} \sim 1$, this means, particles sample in average only one reality per realization. For $q=2$, $R_{n}$ increase logarithmically with step number and in $q=3$ as $\sqrt{n}$.

The sample to sample fluctuations are quantified in terms of the disorder variance of the mean-square displacement between disorder realizations. The self-averaging behavior is probed by the relative variance $\Sigma(t)$. If $\Sigma(t)$ goes asymptotically to zero, $m(t)$ is self-averaging. This does not mean, however, that the fluctuations of $m(t)$ are necessarily small at finite times. We derive explicit expressions for the variance in $q$ dimensions at early and late times, which are compared to TDRW simulations of the full heterogeneous diffusion problem for subdiffusive and diffusive disorder configurations. Under subdiffusive conditions, the mean-square displacement is non self-averaging for $q=1$ and self-averaging for $q \geqslant 2$. Under diffusive conditions, i.e., when $\bar{m}(t) \propto t$, we distinguish scenarios for which the variance of the transition time is infinite and finite. Under both conditions, $m(t)$ is self-averaging in $q$ dimension. The rate of convergence of $\Sigma(t)$ depends on the dimension of space and the heterogeneity distribution.

We study ergodicity in terms of the noise variance of the time-averaged mean-square displacement $m_{a}(t)$, which is based on the definition of the time-averaged particle displacement during a time increment $\Delta$. In a single realization $m_{a}(t) \propto$ $n_{t}$, the number of steps a particle performs to reach time $t$. Due to the sample to sample fluctuations of the diffusion behavior between disorder realization, also the noise variance of $m_{a}(t)$ is a fluctuating quantity. Thus, we probe the ergodicity of $m_{a}(t)$ in terms of the disorder average of its variance, which is dominated by the disorder variance of the step number $n_{t}$. The ergodicity breaking parameter EB is defined as the ratio between the disorder variance of $n_{t}$ and $\bar{n}_{t}^{2}$ consistent with the definition by Bel and Barkai [35] in the framework of CTRW. Ergodicity is studied both for diffusive and subdiffusive disorder configurations. We derive explicit analytical expressions for EB. Under subdiffusive conditions, the mean-square displacement is weakly ergodicity breaking in all dimensions. Under diffusive conditions, for which the mean transition time is finite, 
diffusion is ergodic. The convergence toward ergodicity depends also here on the spatial dimension and disorder distribution.

In conclusion, under diffusive conditions, this means when the mean transition time is finite, the mean-square displacement is both ergodic and self-averaging. Under subdiffusive conditions, on the other hand, the mean-square displacement is weakly ergodicity breaking in all dimensions, while at the same time it is self-averaging for $d>2$. This means, the noise ensemble is not representative of the single-trajectory time statistics, but the disorder ensemble is representative for the noise ensemble in a single-disorder realization.

\section{ACKNOWLEDGMENTS}

M.D. acknowledges the funding from the European Research Council through the project MHetScale (Grant Agreement No. 617511). P.G. acknowledges funding from the European Union's Horizon 2020 Research and Innovation Programme FRACRISK (Grant Agreement No. 640979).

\section{APPENDIX A: EQUIVALENT CTRW FOR $q>2$ SPATIAL DIMENSIONS}

One difference between the TDRW and the CTRW arises from the correlation of successive time increments. Here we re-sample the trajectories in Eq. (13) such that the trajectories are constituted by series of $S_{n}$ independent rescaled space and time increments as

$$
\mathbf{x}_{S_{n}}=\sum_{i=1}^{S_{n}} \hat{\ell}_{n} \boldsymbol{\eta}_{i}, \quad t_{S_{n}}=\sum_{i=1}^{S_{n}} \frac{n}{S_{n}} \tau_{i} .
$$

We require that the variance of $\mathbf{x}_{S_{n}}$ be equal to the variance of $\mathbf{x}_{n}$, which implies

$$
\hat{\ell}_{n}^{2} S_{n} \equiv \ell^{2} .
$$

Thus, we obtain directly $\hat{\ell}_{n}=\sqrt{n / S_{n}} \ell$. This gives for Eq. (A1)

$$
\mathbf{x}_{S_{n}}=\sum_{i=1}^{S_{n}} \sqrt{\frac{n}{S_{n}}} \ell \boldsymbol{\eta}_{i}, \quad t_{S_{n}}=\sum_{i=1}^{S_{n}} \frac{n}{S_{n}} \tau_{i} .
$$

To obtain Eq. (27) for $q>2$, we substitute $S_{n}=b n$ in Eq. (A3) and set $N=\lfloor b n\rfloor$, where $\lfloor\cdot\rfloor$ is the floor function.

\section{APPENDIX B: DISORDER VARIANCE OF MEAN-SQUARE DISPLACEMENT}

The disorder variance of the mean-square displacement is defined by Eq. (39). In the following, we derive expression Eq. (41). To this end, we consider the second disorder moment of $m(t)$. We obtain by using Eq. (20),

$$
\begin{aligned}
\overline{m(t)^{2}}= & \sum_{n, k=0}^{\infty} n k \ell^{4} \frac{1}{R_{n}} \frac{1}{R_{k}} \\
& \times \sum_{i=1}^{R_{n}} \sum_{j=1}^{R_{k}} \overline{\mathbb{I}\left[t_{n}^{(i)} \leqslant t<t_{n+1}^{(i)}\right] \mathbb{I}\left[t_{k}^{(j)} \leqslant t<t_{k+1}^{(j)}\right]} .
\end{aligned}
$$

By definition, the $t_{n}^{(i)}$ and $t_{k}^{(j)}$ are independent for $i \neq j$. For $i=j$ we have by virtue of the impulse functions that $n=k$.
Thus, we can write $\overline{m(t)^{2}}$ as

$$
\overline{m(t)^{2}}=\ell^{4} \sum_{n=0}^{\infty} \frac{n^{2}}{R_{n}} p_{n}(t)+\Lambda(t),
$$

where we defined

$$
\Lambda(t)=\sum_{n, k=0}^{\infty} n k \ell^{4} \frac{1}{R_{n}} \frac{1}{R_{k}} \sum_{i=1}^{R_{n}} \sum_{j=1, j \neq i}^{R_{k}} p_{n}(t) p_{k}(t) .
$$

We used here definition Eq. (26) of the number of steps needed to arrive at time $t . \Lambda(t)$ can be written as

$$
\begin{aligned}
\Lambda(t)= & \sum_{n \geqslant k}^{\infty} n k \ell^{4}\left(1-\frac{1}{R_{n}}\right) p_{n}(t) p_{k}(t) \\
& +\sum_{n<k}^{\infty} n k \ell^{4}\left(1-\frac{1}{R_{k}}\right) p_{n}(t) p_{k}(t),
\end{aligned}
$$

and due to symmetry, we obtain more compactly

$$
\Lambda(t)=\sum_{n, k=1}^{\infty} n k \ell^{4}\left(1-\frac{1}{R_{n}}\right) p_{n}(t) p_{k}(t) .
$$

By comparison with Eq. (30), we identify

$$
\begin{aligned}
\Lambda(t) & =\bar{m}(t)^{2}-\bar{m}(t) \ell^{2} \sum_{n=1}^{\infty} \frac{n}{R_{n}} p_{n}(t) \\
& \equiv \bar{m}(t)^{2}-\bar{m}(t) \ell^{2} \overline{n_{t} / R_{n_{t}}} .
\end{aligned}
$$

Inserting the latter into Eq. (B2) gives

$$
\overline{m(t)^{2}}=\overline{m(t)}^{2}+\ell^{4}\left(\overline{n_{t}^{2} / R_{n_{t}}}-\overline{n_{t}} \overline{n_{t} / R_{n_{t}}}\right),
$$

where we note that

$$
\overline{n_{t}^{2} / R_{n_{t}}}=\sum_{n=0}^{\infty} \frac{n^{2}}{R_{n}} p_{n}(t) .
$$

and that $\bar{m}(t)=\ell^{2} \overline{n_{t}}$; see Eq. (30). Using Eq. (B7) in Eq. (39) for the variance of the mean-square displacement gives Eq. (41).

\section{APPENDIX C: DISTRIBUTION OF NUMBER $\boldsymbol{n}_{t}$ OF RANDOM WALK STEPS}

To determine the long-time behaviors of the disorder average mean-square displacement, its variance and the variance of the time-averaged mean-square displacement, we need to determine averages of function of the number $n_{t}$ of random walk steps. Its distribution is given by

$$
p_{n}(t)=\overline{\delta_{n, n_{t}}}=\overline{\mathbb{I}\left(t_{n} \leqslant t<t_{n+1}\right)} .
$$

Its Laplace transform is given by

$$
p_{n}^{*}(\lambda)=\lambda^{-1} \overline{\left[\exp \left(-\lambda t_{n}\right)-\exp \left(-\lambda t_{n+1}\right)\right]},
$$

where the times $t_{n}$ and $t_{n+1}$ here are

$$
t_{n}=\sum_{i=1}^{S_{n}} \gamma_{n} \tau_{i}, \quad t_{n+1}=\sum_{i=1}^{S_{n+1}} \gamma_{n+1} \tau_{i} .
$$


We defined for convenience $\gamma_{n} \equiv n / S_{n}$. Using these expressions in Eq. (C2) and performing the disorder average, we obtain

$$
p_{n}^{*}(\lambda)=\lambda^{-1}\left[f_{n}^{*}(\lambda)-f_{n+1}^{*}(\lambda)\right] \approx-\lambda^{-1} \frac{d}{d n} f_{n}^{*}(\lambda),
$$

where we defined

$$
f_{n}^{*}(\lambda) \equiv \psi\left(\lambda \gamma_{n}\right)^{S_{n}} .
$$

We consider here transition time PDFs $\psi(t)$ characterized by the power-law behavior Eq. (29) as well as distributions for which all moments exist. We distinguish the cases $0<\beta<1$, $1<\beta<2$ and $\beta>2$ in Eq. (29). The latter covers the leading behavior expected if all moments of $\tau$ exist.

\section{Infinite mean transition time: $0<\beta<1$}

The Laplace transform of $\psi(t)$ can be expanded for small $\lambda$ as

$$
\psi^{*}(\lambda)=1-a_{\beta} \lambda^{\beta}+\cdots,
$$

where $a_{\beta}$ a constant that depends on the specific form of $\psi(t)$. Inserting Eqs. (C6) into (C4) gives for $\lambda \tau_{\kappa} \ll 1$,

$$
f_{n}^{*}(\lambda) \approx \exp \left(-a_{\beta} \alpha_{n} \lambda^{\beta}\right)
$$

where we defined for convenience $\alpha_{n}=\gamma_{n}^{\beta} S_{n}$.

\section{Finite mean transition time: $\beta>1$}

For $1<\beta<2$, this means, if the mean transition time is finite $\langle\tau\rangle<\infty$ and $\left\langle\tau^{2}\right\rangle=\infty$, the Laplace transform of $\psi(t)$ can be expanded as

$$
\psi^{*}(\lambda)=1-\langle\tau\rangle \lambda+b_{\beta} \lambda^{\beta}+\cdots .
$$

where $b_{\beta}$ depends on the specific shape of $\psi(t)$. Inserting Eq. (C8) into Eq. (C4) gives

$$
f_{n}^{*}(\lambda)=\psi^{*}\left(\lambda \gamma_{n}\right)^{S_{n}} \approx \exp (-n \lambda\langle\tau\rangle)\left(1+b_{\beta} \alpha_{n} \lambda^{\beta}+\cdots\right),
$$

where the dots denote sub-leading contributions.

For $\beta>2$, this means, if both the mean and mean-squared transition time are finite, the expansion of $\psi^{*}(\lambda)$ is

$$
\psi^{*}(\lambda)=1-\langle\tau\rangle \lambda+\frac{\left\langle\tau^{2}\right\rangle}{2} \lambda^{2}+\cdots,
$$

where the dots denote sub-leading contributions. Inserting Eq. (C10) into Eq. (C4) gives

$$
f_{n}(\lambda) \equiv \psi\left(\lambda \gamma_{n}\right)^{S_{n}} \approx \exp (-n \lambda\langle\tau\rangle)\left(1+\frac{n^{2}}{S_{n}}\left\langle\tau^{2}\right\rangle \lambda^{2}+\cdots\right),
$$

where the dots denote sub-leading contributions. This expansion is valid for all transition time PDFs, for which the first and second moments exist.

\section{APPENDIX D: DISORDER MOMENTS OF NUMBER $n_{t}$ OF RANDOM WALK STEPS}

The $k$ th disorder moment of the step number $n_{t}$ is defined by

$$
h_{k}(t)=\sum_{n=0}^{\infty} n^{k} p_{n}(t)
$$

The Laplace transform of $h_{k}(t)$ can with the above definitions be approximated as

$$
h_{k}^{*}(\lambda) \approx-\lambda^{-1} \int_{0}^{\infty} d n n^{k} \frac{d}{d n} f_{n}^{*}(\lambda) .
$$

Integration by parts gives

$$
h_{k}^{*}(\lambda) \approx k \lambda^{-1} \int_{0}^{\infty} d n n^{k-1} f_{n}^{*}(\lambda) .
$$

\section{Infinite mean transition time: $0<\beta<1$}

We write Eq. (D3) by using Eq. (C7) as

$$
h_{k}^{*}(\lambda) \approx k \lambda^{-1} \int_{0}^{\infty} q n n^{k-1} \exp \left(-a_{\beta} \alpha_{n} \lambda^{\beta}\right) .
$$

\section{a. Spatial dimension $q=1$}

In $q=1, S_{n} \sim n^{q / 2}$ and $\gamma_{n}=n^{1-q / 2}$, therefore, $\alpha_{n}=$ $n^{\frac{2 \beta-d \beta+d}{2}}$. We obtain by rescaling $n$,

$$
h_{k}^{*}(\lambda) \approx \lambda^{-1-\frac{2 \alpha \beta}{2 \beta-\alpha \beta+d}} k \int_{0}^{\infty} q n n^{k-1} \exp \left(-a_{\beta} n^{\frac{2 \beta-\alpha \beta+d}{2}}\right) .
$$

Inverse Laplace transform gives

$$
h_{k}(t) \propto t^{\frac{2 k \beta}{2 \beta-\beta \beta+d}} .
$$

\section{b. Spatial dimension $q=2$}

In $q=2, S_{n} \sim n / \ln (n)$ and $\gamma_{n}=\ln (n)$, therefore, $\alpha_{n}=$ $n \ln (n)^{\beta-1}$, such that

$$
h_{k}^{*}(\lambda) \approx \lambda^{-1} k \int_{0}^{\infty} q n n^{k-1} \exp \left[-a_{\beta} \lambda^{\beta} n \ln (n)^{\beta-1}\right] .
$$

We rescale now $n \rightarrow n \lambda^{\beta} \ln (1 / \lambda)^{\beta-1}$. Thus, we obtain in leading order in the limit $\lambda \rightarrow 0$,

$$
h_{k}^{*}(\lambda) \approx \lambda^{-1-k \beta} \ln (1 / \lambda)^{k-k \beta} k \int_{0}^{\infty} q n n^{k-1} \exp \left(-a_{\beta} \beta^{\beta} n\right) .
$$

Inverse Laplace transform gives

$$
h_{k}(t) \propto t^{k \beta} \ln (t)^{k-k \beta} .
$$

\section{c. Spatial dimension $q>2$}

In $q>2, S_{n} \sim n$ and $\gamma_{n}=1$, therefore, $\alpha_{n}=n$, such that

$$
h_{k}^{*}(\lambda) \approx \lambda^{-1-\beta} k \int_{0}^{\infty} d n n^{k-1} \exp \left(-a_{\beta} n\right) .
$$

Inverse Laplace transform gives

$$
h_{k}(t) \propto t^{k \beta} .
$$




\section{Finite mean transition time $\beta>1$}

For $1<\beta<1$, we use Eq. (C9) in Eq. (C4) in the Laplace transform of Eq. (30) to obtain

$$
\begin{aligned}
h_{k}^{*}(\lambda) \approx & \lambda^{-1} k \int_{0}^{\infty} d n n^{k-1} \exp (-\lambda n \bar{\tau}) \\
& +\lambda^{-1} k \int_{0}^{\infty} d n n^{k-1} \exp (-\lambda n \bar{\tau}) b_{\beta} \alpha_{n} \lambda^{\beta}+\cdots
\end{aligned}
$$

The case $\beta>2$ is obtained according to Eq. (C11) by setting $\beta=2$ and $b_{\beta}=b_{2}=\left\langle\tau^{2}\right\rangle / 2$.

\section{a. $q=1$}

Using $\alpha_{n}=n^{\frac{2 \beta-d \beta+d}{2}}$ and rescaling of $n$, we obtain

$$
\begin{aligned}
h_{k}^{*}(\lambda) \approx & \lambda^{-1-k} k \int_{0}^{\infty} d n n^{k-1} \exp (-n \bar{\tau}) \\
& +\lambda^{-1-k-\frac{d(1-\beta)}{2}} k \int_{0}^{\infty} d n n^{k-1+\frac{2 \beta-d \beta+d}{2}} \exp (-n \bar{\tau}) b_{\beta} .
\end{aligned}
$$

Inverse Laplace transform gives

$$
h_{k}(t)=(t / \bar{\tau})^{k}+A_{1}(t /\langle\tau\rangle)^{k+\frac{d(1-\beta)}{2}},
$$

where we defined

$$
\begin{gathered}
A_{1}=\frac{b_{\beta}}{\bar{\tau}^{\beta}} \frac{\Gamma\left(k+\frac{2 \beta-d \beta+d}{2}\right)}{\Gamma\left[k+1+\frac{d(1-\beta)}{2}\right]} . \\
\text { b. } \boldsymbol{q}=\mathbf{2}
\end{gathered}
$$

Using $\alpha_{n}=n \ln (n)^{\beta-1}$ and rescaling of $n$, we obtain in leading order

$$
\begin{aligned}
h_{k}^{*}(\lambda) \approx & \lambda^{-1-k} k \int_{0}^{\infty} d n n^{k-1} \exp (-n \bar{\tau}) \\
& +\lambda^{-2-k+\beta} \ln (1 / \lambda)^{\beta-1} k \int_{0}^{\infty} d n n^{k} \exp (-n \bar{\tau}) b_{\beta} .
\end{aligned}
$$

Inverse Laplace transform gives

$$
h_{k}(t)=(t / \bar{\tau})^{k}+A_{2}(t / \bar{\tau})^{1+k-\beta} \ln (t / \bar{\tau})^{\beta-1},
$$

where we defined

$$
\begin{gathered}
A_{2}=\frac{b_{\beta}}{\bar{\tau}^{\beta}} \frac{k \Gamma(k+1)}{\Gamma(k+2-\beta)} . \\
\text { c. } \boldsymbol{q}>\mathbf{2}
\end{gathered}
$$

Inverse Laplace transform gives

$$
h_{k}(t)=(t / \bar{\tau})^{k}+A_{2}(t /\langle\tau\rangle)^{1+k-\beta} .
$$

\section{APPENDIX E: VARIANCE OF THE MEAN-SQUARE DISPLACEMENT}

To determine the behavior of the variance $\sigma_{m}^{2}(t)$ of the mean-square displacement $m(t)$, we need to evaluate Eq. (41), which involves the disorder moments $\overline{n_{t}^{2} / R_{n_{t}}}$ and $\overline{n_{t} / R_{n_{t}}}$ of the random step number $n_{t}$.

\section{Spatial dimension $q=1$}

For $q=1, R_{n}=2^{q / 2}$. Thus, we obtain for $\sigma_{m}^{2}(t)$,

$$
\sigma_{m}^{2}(t)=\frac{\ell^{4}}{2^{q / 2}}\left(\overline{n_{t}^{2}}-{\overline{n_{t}}}^{2}\right)=\frac{\ell^{4}}{2^{q / 2}}\left[h_{2}(t)-h_{1}(t)^{2}\right] .
$$

a. Mean transition time infinite: $0<\beta<1$

We obtain from the previous section that

$$
\sigma_{m}^{2}(t) \propto t^{\frac{4 \beta}{2 \beta-d \beta+d}} .
$$

\section{b. Mean transition time finite: $\beta>1$}

For $1<\beta<2$, we obtain from the previous section

$$
\sigma_{m}^{2}(t) \propto(t / \bar{\tau})^{2+\frac{q(1-\beta)}{2}} .
$$

This means, for $\beta>2$, that

$$
\sigma_{m}^{2}(t) \propto(t / \bar{\tau})^{2-\frac{q}{2}} .
$$

\section{Spatial dimension $q=2$}

For $q=2, R_{n}=2 \ln (n)$. Thus, we obtain for $\sigma_{m}^{2}(t)$,

$$
\sigma_{m}^{2}(t)=\ell^{4}\left[\overline{n_{t}^{2} / \ln \left(n_{t}\right)}-\overline{n_{t} / \ln \left(n_{t}\right)} \overline{n_{t}}\right] .
$$

This means we need to determine

$$
J_{k}(t)=\sum_{n=0}^{\infty} \frac{n^{k}}{\ln (n)} p_{n}(t)
$$

for $k=1,2$. We obtain from Eq. (C4) for its Laplace transform,

$$
J_{k}^{*}(\lambda) \approx-\lambda^{-1} \int_{0}^{\infty} d n \frac{n^{k}}{\ln (n)} \frac{d}{d n} f_{n}^{*}(\lambda)
$$

Integration by parts gives

$$
J_{k}^{*}(\lambda) \approx \lambda^{-1} \int_{0}^{\infty} d n n^{k-1}\left[\frac{k}{\ln (n)}-\frac{1}{\ln (n)^{2}}\right] f_{n}^{*}(\lambda) .
$$

\section{a. Infinite mean transition time: $0<\beta<1$}

We obtain by using Eq. (C7) for $\alpha_{n}=n \ln (n)^{\beta-1}$,

$$
\begin{aligned}
J_{k}^{*}(\lambda) \approx & \lambda^{-1} \int_{0}^{\infty} d n \frac{k n^{k-1}}{\ln (n)}\left[1-\frac{1}{k \ln (n)}\right] \\
& \times \exp \left[-a_{\beta} \lambda^{\beta} n \ln (n)^{\beta-1}\right] .
\end{aligned}
$$

Rescaling $n \rightarrow n \lambda^{\beta} \ln (1 / \lambda)^{\beta-1}$, we obtain in leading order in the limit $\lambda \rightarrow 0$,

$$
J_{k}^{*}(\lambda) \approx \lambda^{-1-k \beta} \ln (1 / \lambda)^{k-1-k \beta} \int_{0}^{\infty} d n k n^{k-1} \exp \left(-a_{\beta} \beta^{\beta} n\right) .
$$


Inverse Laplace transform gives

$$
J_{k}(t) \propto t^{k \beta} \ln (t)^{k-1-k \beta} .
$$

Thus, we obtain for $\sigma_{m}^{2}(t)$ the scaling

$$
\sigma_{m}^{2}(t) \propto t^{2 \beta} \ln (t)^{1-2 \beta} .
$$

\section{b. Finite mean transition time: $\beta>1$}

We obtain by using Eq. (C9) for $\alpha_{n}=n \ln (n)^{\beta-1}$,

$$
\begin{aligned}
J_{k}^{*}(\lambda) \approx & \lambda^{-1} \int_{0}^{\infty} d n \frac{k n^{k-1}}{\ln (n)}\left[1-\frac{1}{k \ln (n)}\right] \exp (-n \lambda \bar{\tau}) \\
& +\lambda^{-1+\beta} \int_{0}^{\infty} d n k n^{k} \ln (n)^{\beta-2}\left[1-\frac{1}{k \ln (n)}\right] \\
& \times \exp (-n \lambda \bar{\tau}) b_{\beta} .
\end{aligned}
$$

Rescaling of $n \rightarrow n \lambda$ gives in leading order

$$
\begin{aligned}
J_{k}^{*}(\lambda) \approx & \frac{\lambda^{-1-k}}{\ln (1 / \lambda)} \int_{0}^{\infty} d n k n^{k-1} \exp (-n \bar{\tau}) \\
& +\lambda^{-2-k+\beta} \ln (1 / \lambda)^{\beta-2} \int_{0}^{\infty} d n k n^{k} \exp (-n \bar{\tau}) b_{\beta} .
\end{aligned}
$$

Inverse Laplace transform gives

$$
J_{k}(t) \approx \frac{(t / \bar{\tau})^{k}}{\ln (t /\langle\tau\rangle)}+A_{2}(t / \bar{\tau})^{1+k-\beta} \ln (t / \bar{\tau})^{\beta-2} .
$$

Thus, we obtain for $\sigma_{m}^{2}(t)$ the behavior

$$
\sigma_{m}^{2}(t) \propto(t / \bar{\tau})^{3-\beta} \ln (t / \bar{\tau})^{\beta-2} .
$$

For $\beta>2$, we obtain

$$
J_{k}(t) \approx \frac{(t / \bar{\tau})^{k}}{\ln (t /\langle\tau\rangle)}+A_{2}(t / \bar{\tau})^{k-1}
$$

and

$$
\sigma_{m}^{2}(t) \propto t / \bar{\tau}
$$

\section{Spatial dimension $q>2$}

For $q>2, R_{n} \sim 2^{q / 2} n^{q / 2-1}$. Thus, we obtain for $\sigma_{m}^{2}(t)$ from Eq. (41)

$$
\begin{aligned}
\sigma_{m}^{2}(t) & =\frac{\ell^{4}}{2^{q / 2}}\left(\overline{n_{t}^{3-q / 2}}-\overline{n_{t}^{2-q / 2}} \overline{n_{t}}\right) \\
& =\ell^{4}\left[h_{3-q / 2}(t)-h_{2-q / 2}(t) h_{1}(t)\right] .
\end{aligned}
$$

\section{a. Infinite mean transition time: $0<\beta<1$}

Using the results for the $h_{k}(t)$ from the previous section, we obtain

$$
\sigma_{m}^{2}(t) \propto t^{\beta(3-q / 2)}
$$

\section{b. Finite mean transition time: $\beta>1$}

Along the same lines, we obtain for $1<\beta<2$

$$
\sigma_{m}^{2}(t) \propto t^{4-\beta-q / 2} \text {. }
$$

For $\beta>2$, we obtain accordingly

$$
\sigma_{m}^{2}(t) \propto t^{2-q / 2} .
$$

\section{APPENDIX F: NUMERICAL SIMULATIONS}

The numerical simulations use a TDRW algorithm [44]. The TDRW approach is based on constant length displacement of particles described by the recursive relations:

$$
\mathbf{x}_{i}(n+1)=\mathbf{x}_{j}(n)+\boldsymbol{\xi}_{i j}, \quad t(n+1)=t(n)+\tau_{j} .
$$

The transition probability $w_{i j}$ to move from voxel $j$ to voxel $i$ for a transition of length $\left|\boldsymbol{\xi}_{i j}\right|$ is given by $w_{i j}=1 /(2 q)$ and the transition time associated to voxel $j$ is $\tau_{j}=\theta_{j} \ell^{2} /(2 q \kappa)$. In the case of the annealed disorder model, the random variable $\theta_{j}$ is not linked to the voxel $j$, but on the $n$th step, thus $\theta_{j}=\theta_{n}$ and consequently $\tau_{j}=\tau_{n}$.

For the numerical simulations, we consider the Pareto distribution,

$$
p_{\theta}(\theta)=\beta \theta^{-1-\beta} H(\theta-1),
$$

with $H(\cdot)$ the Heaviside step function. We obtain according to Eq. (12) the following distribution of transition times:

$$
\psi(\tau)=\frac{\beta}{\tau_{\kappa}}\left(\frac{\tau}{\tau_{\kappa}}\right)^{-1-\beta} \gamma\left(1+\beta, \tau / \tau_{\kappa}\right),
$$

where $\gamma(a, z)$ is the lower incomplete Gamma function [53]. The numerical calculations of the disorder average particle distribution use $10^{3}-10^{4}$ particles in each of the $10^{3}-10^{4}$ disorder realizations. The calculations of the particle distribution in single realizations use $10^{5}-10^{7}$ particles. The calculations for average mean-square displacement use $10^{3}-10^{4}$ particles in each of the $10^{4}$ disorder realizations. The calculations for the disorder variance of the mean-square displacement use $10^{5}$ particles in each of the $10^{3}$ realizations. The calculations for the variance of the time-averaged mean-square displacement use $10^{4}$ particles in each of the $10^{3}$ disorder realizations.
[1] J. P. Bouchaud and A. Georges, Phys. Rep. 195, 127 (1990).

[2] S. Condamin, O. Bénichou, V. Tejedor, R. Voituriez, and J. Klafter, Nature 450, 77 (2007).

[3] S. Havlin and D. Ben-Avraham, Adv. Phys. 51, 187 (2002).

[4] J. Klafter and I. Sokolov, Phys. World 18, 29 (2005).

[5] I. M. Sokolov, Soft Matter 8, 9043 (2012).

[6] G. de Marsily, E. Ledoux, A. Barbreau, and J. Margat, Science 197, 519 (1977).

[7] B. Noetinger and T. Estebenet, Transp. Porous Media 39, 315 (2000).
[8] R. Benke and S. Painter, Water Resour. Res. 39, 1324 (2003).

[9] F. Delay, G. Porel, and P. Sardini, C. R. Geoscience 334, 967 (2002).

[10] C. Poinssot and H. Geckeis, eds., Radionuclide Behavior in the Natural Environment (Woodhead Publishing, Sawston, UK, 2012).

[11] J. Nelson, Phys. Rev. B 59, 15374 (1999).

[12] D. Wang, C. He, M. P. Stoykovich, and D. K. Schwartz, ACS Nano 9, 1656 (2015). 
[13] J. Dong, Y. Cheng, Q. Liu, H. Zhang, K. Zhang, and B. Hu, Energy Fuels 31, 2643 (2017).

[14] T. W. Patzek, F. Male, and M. Marder, Proc. Natl. Acad. Sci. U.S.A. 110, 19731 (2013).

[15] K. Falk, B. Coasne, R. Pellenq, F.-J. Ulm, and L. Bocquet, Nat. Commun. 6, 6949 (2015).

[16] A. Russian, P. Gouze, M. Dentz, and A. Gringarten, Transp. Porous Media 109, 109 (2015).

[17] M. Quintard and S. Whitaker, Adv. Heat Transfer 23, 369 (1993).

[18] B. Berkowitz and H. Scher, Phys. Rev. Lett. 79, 4038 (1997).

[19] B. Berkowitz, A. Cortis, M. Dentz, and H. Scher, Rev. Geophys. 44, RG2003 (2006).

[20] T. Le Borgne, M. Dentz, and J. Carrera, Phys. Rev. E 78, 026308 (2008).

[21] M. Klepikova, T. Le Borgne, O. Bour, M. Dentz, R. Hochreutner, and N. Lavenant, Water Resour. Res. 52, 5442 (2016).

[22] H. Scher and M. Lax, Phys. Rev. B 7, 4491 (1973).

[23] P. Barthelemy, J. Bertolotti, and D. S. Wiersma, Nature 453, 495 (2008).

[24] F. Höfling and T. Franosch, Rep. Prog. Phys. 76, 046602 (2013).

[25] B. M. Regner, D. Vucinic, C. Domnisoru, T. M. Bartol, M. W. Hetzer, D. M. Tartakovsky, and T. J. Sejnowski, Biophys. J. 104, 1652 (2013).

[26] P. Massignan, C. Manzo, J. A. Torreno-Pina, M. F. GarcíaParajo, M. Lewenstein, and G. J. Lapeyre, Jr., Phys. Rev. Lett. 112, 150603 (2014).

[27] E. Barkai, Y. Garini, and R. Metzler, Phys. Today 65(8), 29 (2012).

[28] C. Manzo, J. A. Torreno-Pina, P. Massignan, G. J. Lapeyre, Jr., M. Lewenstein, and M. F. García-Parajo, Phys. Rev. X 5, 011021 (2015).

[29] G. Seisenberger, M. U. Ried, T. Endreß, H. Buning, M. Hallek, and C. Brauchle, Science 294, 1929 (2001).

[30] R. Metzler, J. Jae-Hyung, A. Cherstvy, and E. Barkai, Phys. Chem. Chem. Phys. 16, 24128 (2014).

[31] T. Akimoto, E. Barkai, and K. Saito, Phys. Rev. Lett. 117, 180602 (2016).
[32] M. Dentz, A. Russian, and P. Gouze, Phys. Rev. E 93, 010101(R) (2016).

[33] J. P. Bouchaud, J. Phys. I France 2, 1705 (1992).

[34] X. Brokmann, J.-P. Hermier, G. Messin, P. Desbiolles, J. P. Bouchaud, and M. Dahan, Phys. Rev. Lett. 90, 120601 (2003).

[35] G. Bel and E. Barkai, Phys. Rev. Lett. 94, 240602 (2005).

[36] A. Lubelski, I. M. Sokolov, and J. Klafter, Phys. Rev. Lett. 100, 250602 (2008)

[37] Y. Meroz, I. M. Sokolov, and J. Klafter, Phys. Rev. E 81, 010101(R) (2010).

[38] T. Miyaguchi and T. Akimoto, Phys. Rev. E 83, 031926 (2011).

[39] R. Metzler and J. Klafter, Phys. Rep. 339, 1 (2000).

[40] I. M. Sokolov, A. Blumen, and J. Klafter, Physica A (Amsterdam) 302, 268 (2001).

[41] M. Dentz, A. Russian, and P. Gouze, Phys. Rev. E 94, 019902(E) (2016).

[42] E. M. Bertin and J.-P. Bouchaud, Phys. Rev. E. 67, 026128 (2003).

[43] H. Risken, The Fokker-Planck Equation (Springer, Heidelberg/ New York, 1996).

[44] M. Dentz, P. Gouze, A. Russian, J. Dweik, and F. Delay, Adv. Water Resour. 49, 13 (2012).

[45] J. F. McCarthy, J. Phys. A 26, 2495 (1993).

[46] B. Noetinger, D. Roubinet, A. Russian, T. Le Borgne, F. Delay, M. Dentz, J.-R. de Dreuzy, and P. Gouze, Transp. Porous Media 115, 345 (2016).

[47] A. Russian, M. Dentz, and P. Gouze, Water Resour. Res. 52, 3309 (2016).

[48] G. H. Vineyard, J. Math. Phys. 4, 1191 (1963).

[49] C. Aslangul, J.-P. Bouchaud, A. Georges, N. Pottier, and D. Saint-James, J. Stat. Phys. 55, 461 (1989).

[50] S. Wiseman and E. Domany, Phys. Rev. E 52, 3469 (1995).

[51] G. Matheron, Economic Geol. 58, 1246 (1963).

[52] Y. He, S. Burov, R. Metzler, and E. Barkai, Phys. Rev. Lett. 101, 058101 (2008).

[53] M. Abramowitz and I. A. Stegun, Handbook of Mathematical Functions (Dover Publications, New York, 1972). 